Supplementary Information

\title{
Discovery of Stable and Selective Antibody Mimetics from Combinatorial Libraries of Polyvalent, Loop-Functionalized Peptoid Nanosheets
}

Jae Hong Kim,${ }^{\dagger 1}$ Samuel C. Kim, ${ }^{\dagger 2}$ Mark A. Kline, ${ }^{1}$ Elissa M. Grzincic, ${ }^{1}$ Blakely W. Tresca, ${ }^{1}$ Joshua Cardiel, ${ }^{2}$ Mohsen Karbaschi, ${ }^{2}$ Dilani C. Dehigaspitiya, ${ }^{3}$ Yulin Chen, ${ }^{4}$ Venkatareddy Udumula, ${ }^{4}$ Tengyue Jian, ${ }^{4}$ Daniel J. Murray, ${ }^{1}$ Lisa Yun, ${ }^{1}$ Michael D. Connolly ${ }^{1}$, Jianfang Liu, ${ }^{1}$ Gang Ren, ${ }^{1}$ Chun-Long Chen, ${ }^{4}$ Kent Kirshenbaum, ${ }^{3}$ Adam R. Abate*, ${ }^{2,5}$ Ronald N.

$$
\text { Zuckermann*1 }
$$

${ }^{1}$ The Molecular Foundry, Lawrence Berkeley National Laboratory, 1 Cyclotron Road, Berkeley, CA 94720, USA.

${ }^{2}$ Department of Bioengineering and Therapeutic Sciences, University of California, San Francisco, 1700 4th street, San Francisco, CA 94158 USA.

${ }^{3}$ Department of Chemistry, New York University, 100 Washington Square East, New York, NY 10003, USA.

${ }^{4}$ Physical Sciences Division, Pacific Northwest National Laboratory, Richland, WA 99352, USA.

${ }^{5}$ Chan Zuckerberg Biohub, San Francisco, CA 94158, USA.

\section{Table of Contents}

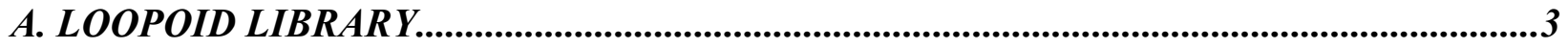

Characterization of free peptoids by analytical HPLC and MALDI-TOF .........................................3

Table S1. HP pattern, sequence, characterization of loopoids ........................................................11

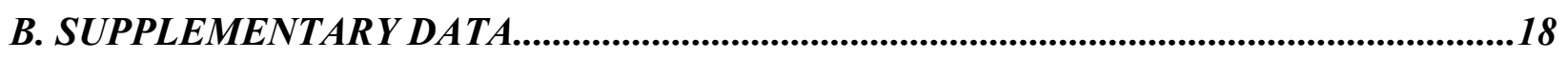

Anthrax protective antigen (PA63)7 preparation.........................................................................................18

Figure S1. Pipet tip geometry for interfacial compression. ..............................................................19

Figure S2. Fluorescence microscopic images of pipet-generated nanosheets...................................20

Figure S3. Homogeneous FRET assay for the identification of binding affinity of loopoid nanosheet. ..........................................................................................................................................21

Figure S4. FRET assay to identify protein binding to peptoid nanosheet........................................22 
Figure S5. Identification of the origin of FRET signal by confocal microscopy...............................23

Figure S6. Curve fitting method provides more accurate measure of FRET efficiency..................24

Figure S7. Z-factor calculation validates the reliability of developed FRET assay..........................25

Figure S8. Validation of binding specificity of L034 and TYWWLD nanosheet by FRET assay..26

Figure S9. Validation of binding specificity of TYWWLD nanosheet by fluorescent microscopy.

Table S2. Comparison of HP pattern and chemical property of monomers between L034 and TYWWLD loop sequences.......................................................................................................................28

Figure S10. FRET-based validation of protein binding to nanosheets classified as PHHHHP

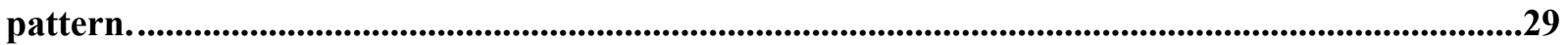

Figure S11. Chemical structure and (PA63) 7 binding specificity of Rtoid. .......................................30

Table S3. Binding parameters of B36, TYWWLD, and L034 nanosheet measured by bio-layer

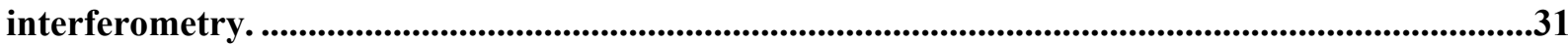




\section{A. LOOPOID LIBRARY}

\section{Characterization of free peptoids by analytical HPLC and MALDI-TOF}

Several representative peptoid strands are shown in this section.

TYWWLD-inserted loopoid strand

2: UV Detector: $214 \mathrm{Nm}$

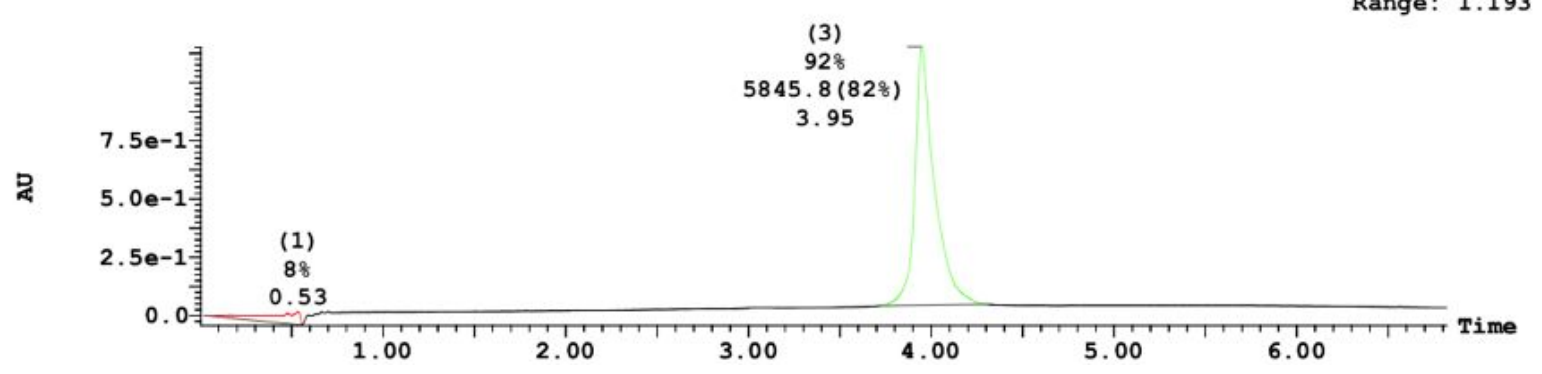

TOF/TOF'M Linear Spec \#1 MC[BP = 5839.0, 3561]

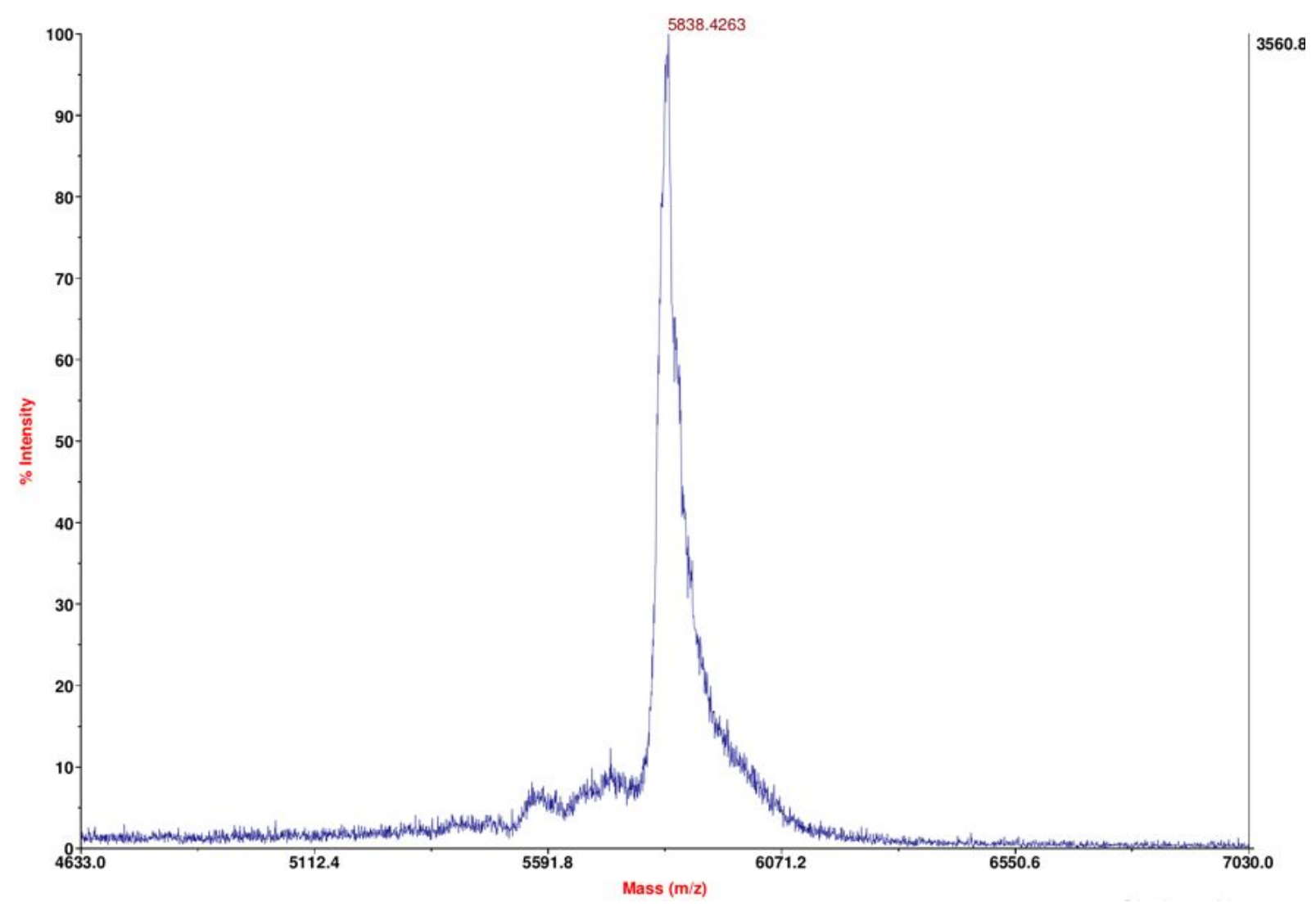

UPLC (5-95\% $6.8 \mathrm{~min}) \mathrm{RT}=3.95 \mathrm{~min}$.

MALDI-TOF calc. $(\mathrm{M}+\mathrm{H})^{+}=5847.78$, obs. $=5838.43$ 
TYWWLD-inspired loopoid strand (Rtoid)
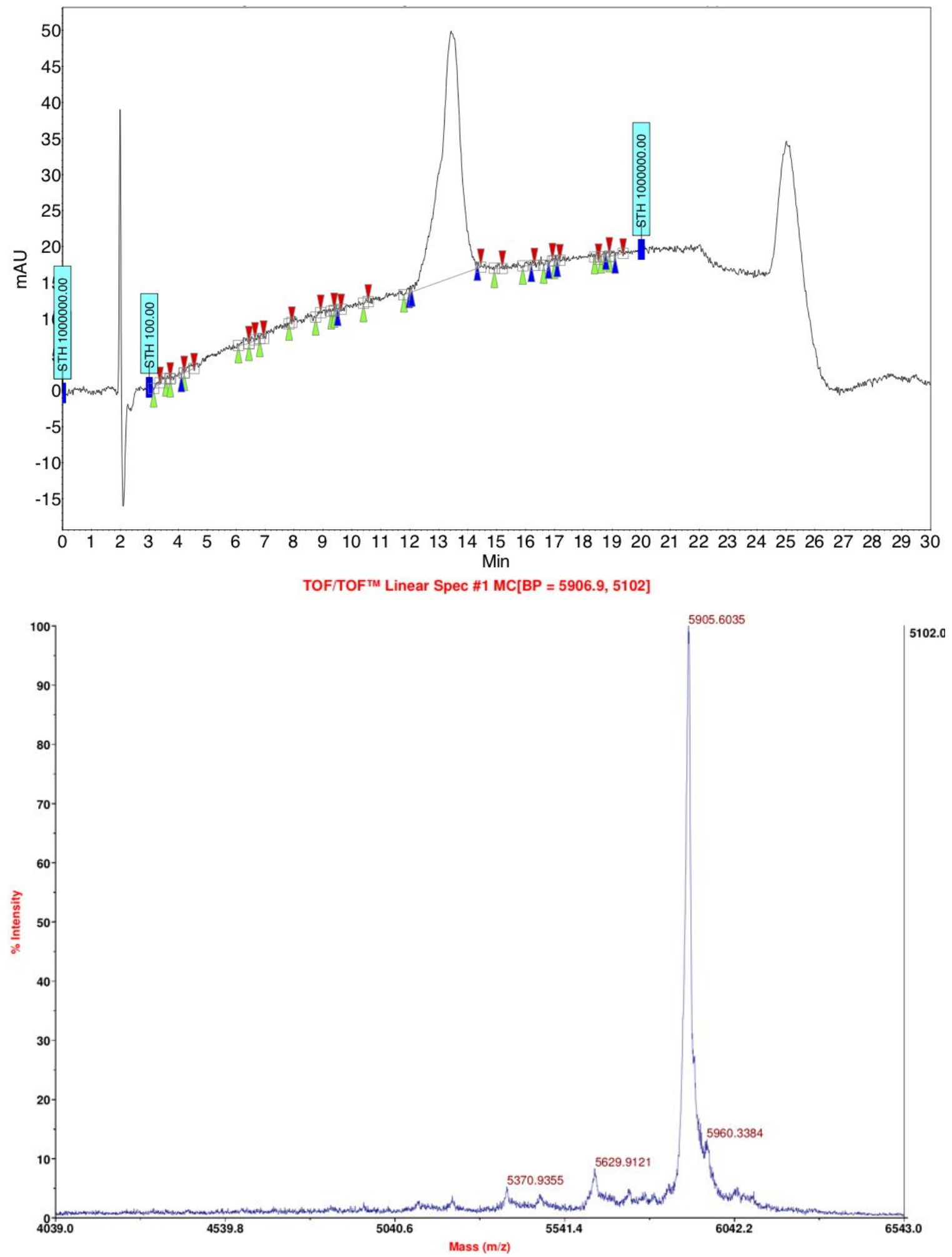

HPLC (5-95\% $20 \mathrm{~min}) \mathrm{RT}=13.44 \mathrm{~min}$

MALDI-TOF calc. $(\mathrm{M}+\mathrm{H})^{+}=5917.92$, obs. $=5905.60$ 


\section{L002 loopoid strand}

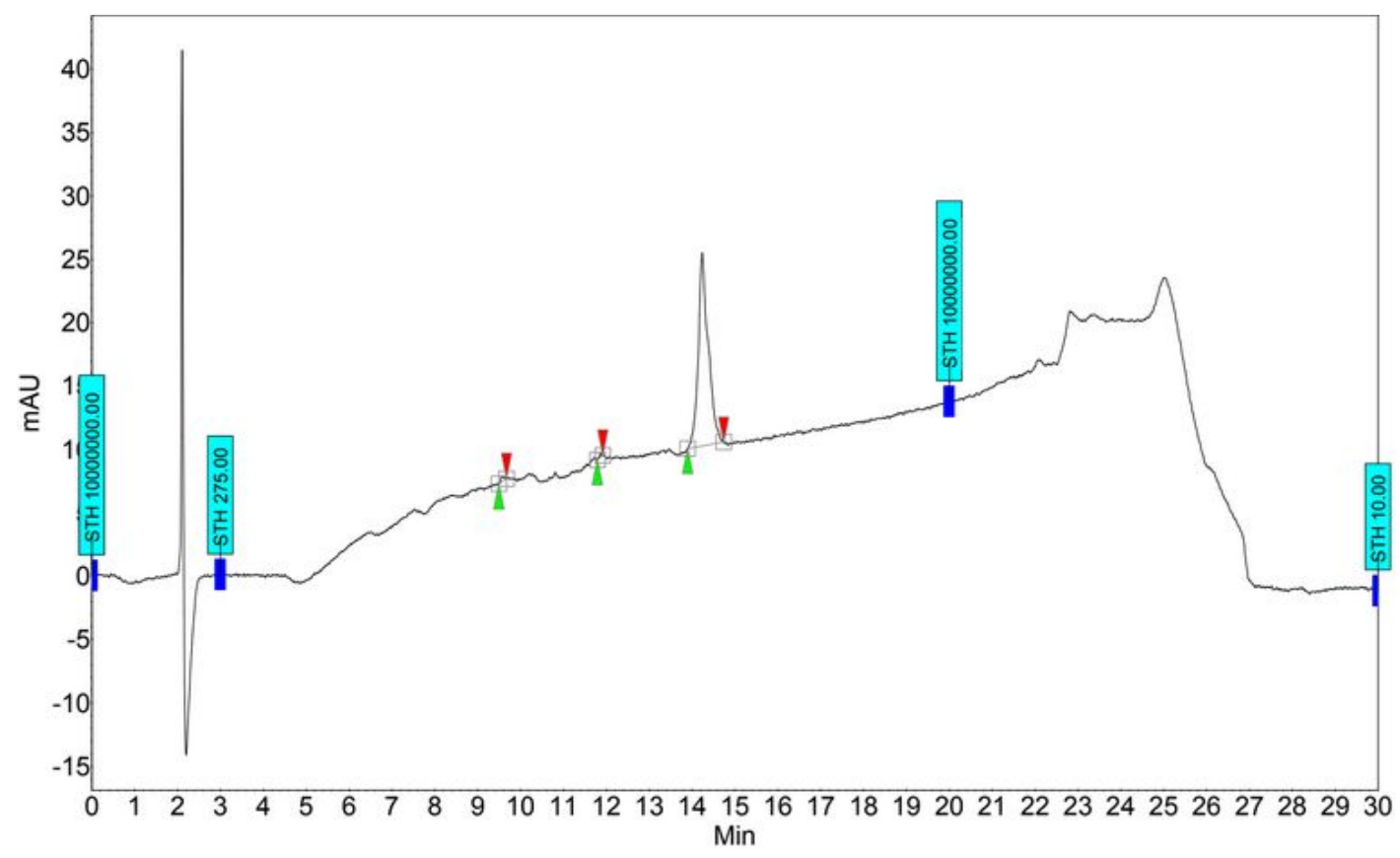

TOF $/$ TOF Tw Linear Spec \#1 MC[BP = 5848.1, 90]

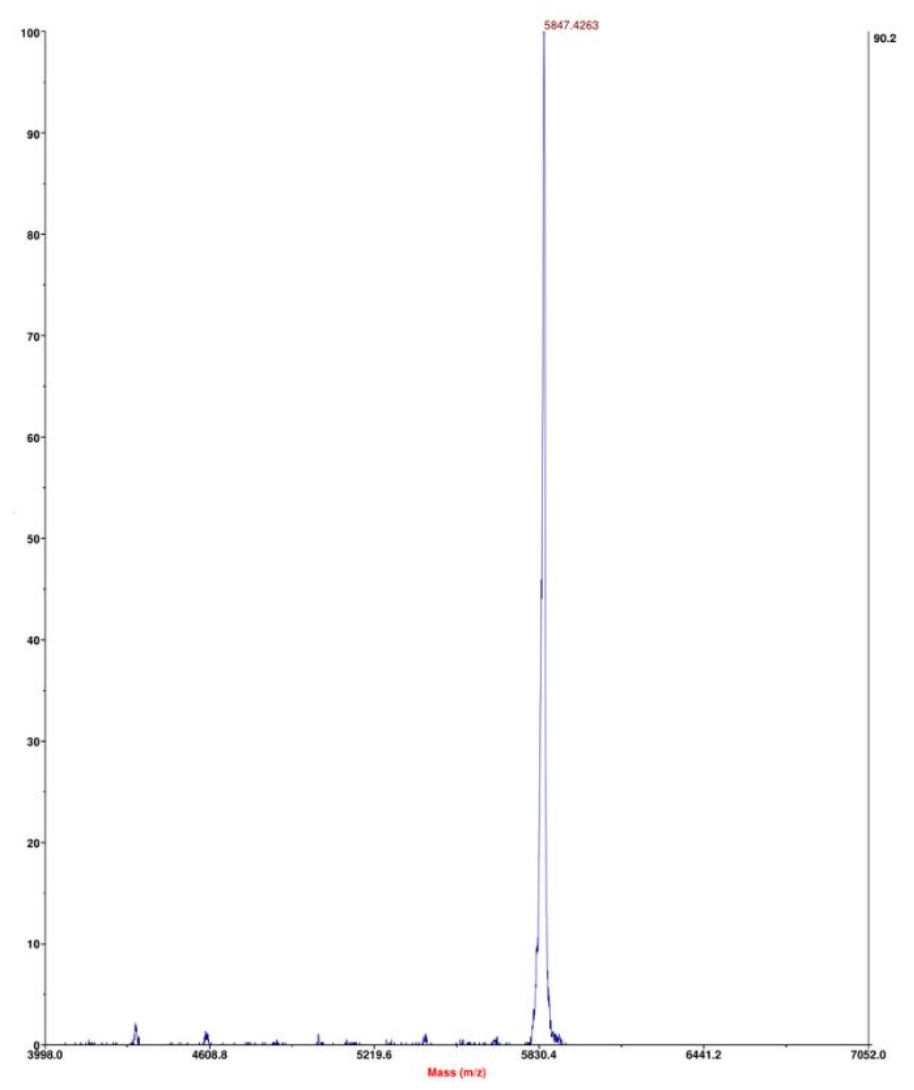

HPLC (5-95\% $20 \mathrm{~min}) \mathrm{RT}=14.23 \mathrm{~min}$

MALDI-TOF calc. $(\mathrm{M}+\mathrm{H})^{+} 5855.85$, obs. $=5848.1$ 


\section{L006 loopoid strand}
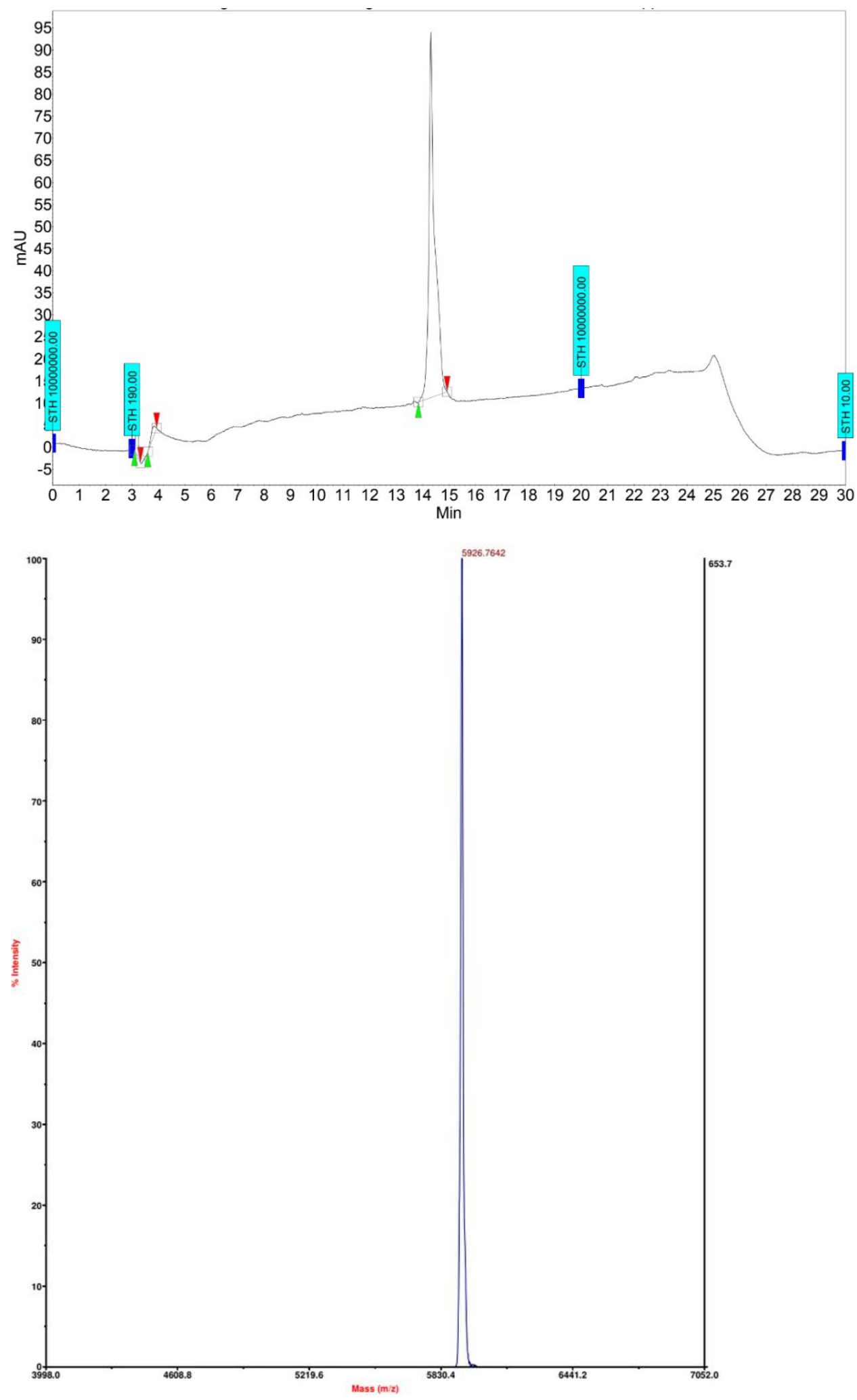

HPLC (5-95\% $20 \mathrm{~min}) \mathrm{RT}=14.31 \mathrm{~min}$

MALDI-TOF calc. $(\mathrm{M}+\mathrm{H})^{+} 5932.93$, obs. $=5927.8$ 


\section{L009 loopoid strand}

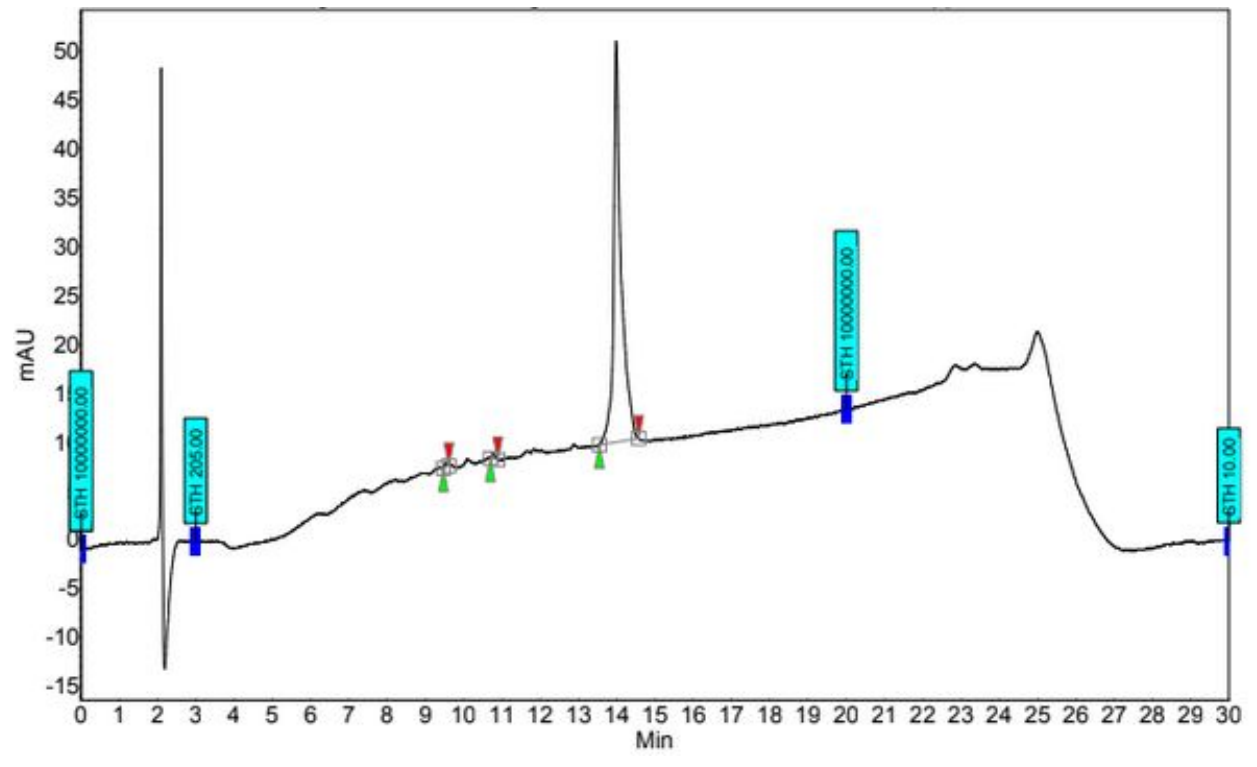

TOF $/$ TOF ${ }^{w}$ Linear Spec $\# 1$ MC[BP = 5705.6, 2592]

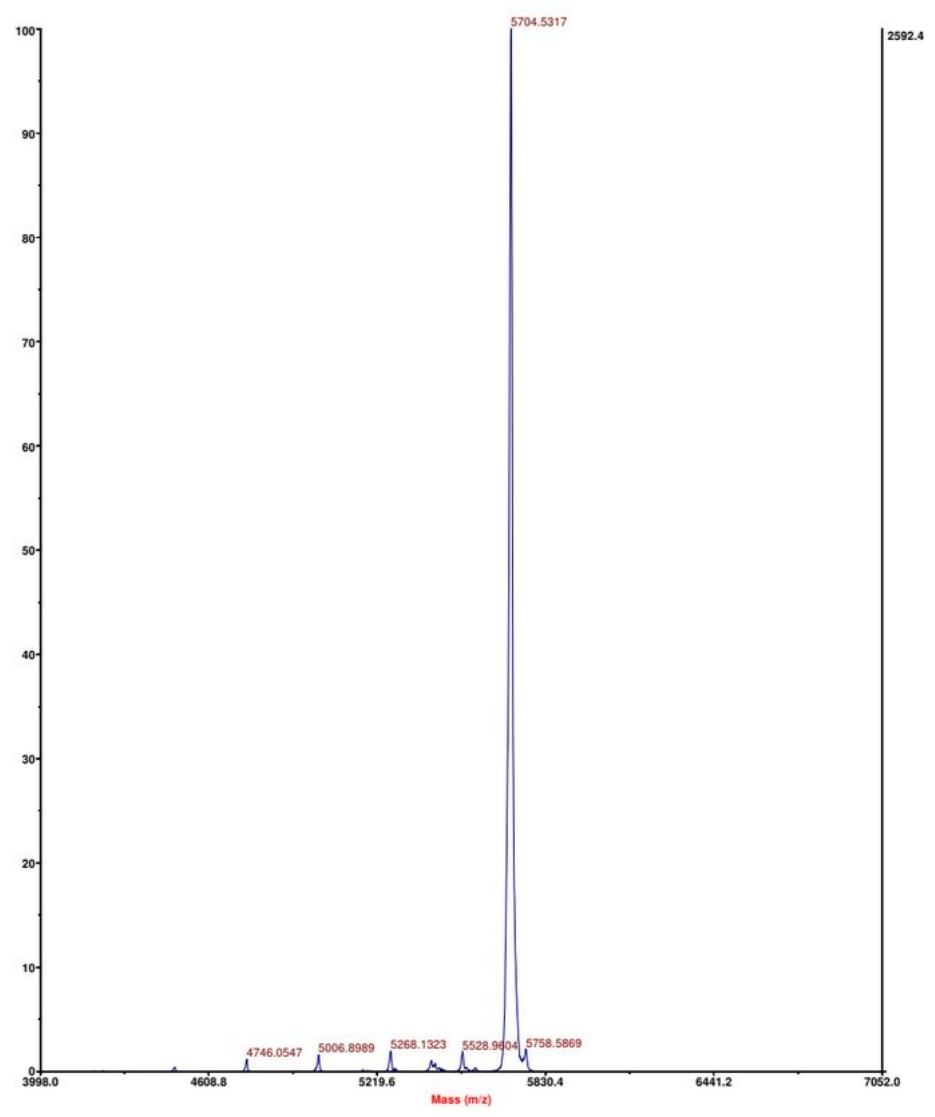

HPLC (5-95\% 20 min) RT $=13.99 \mathrm{~min}$

MALDI-TOF calc. $(\mathrm{M}+\mathrm{H})^{+} 5710.64$, obs. $=5705.6$ 


\section{L011 loopoid strand}
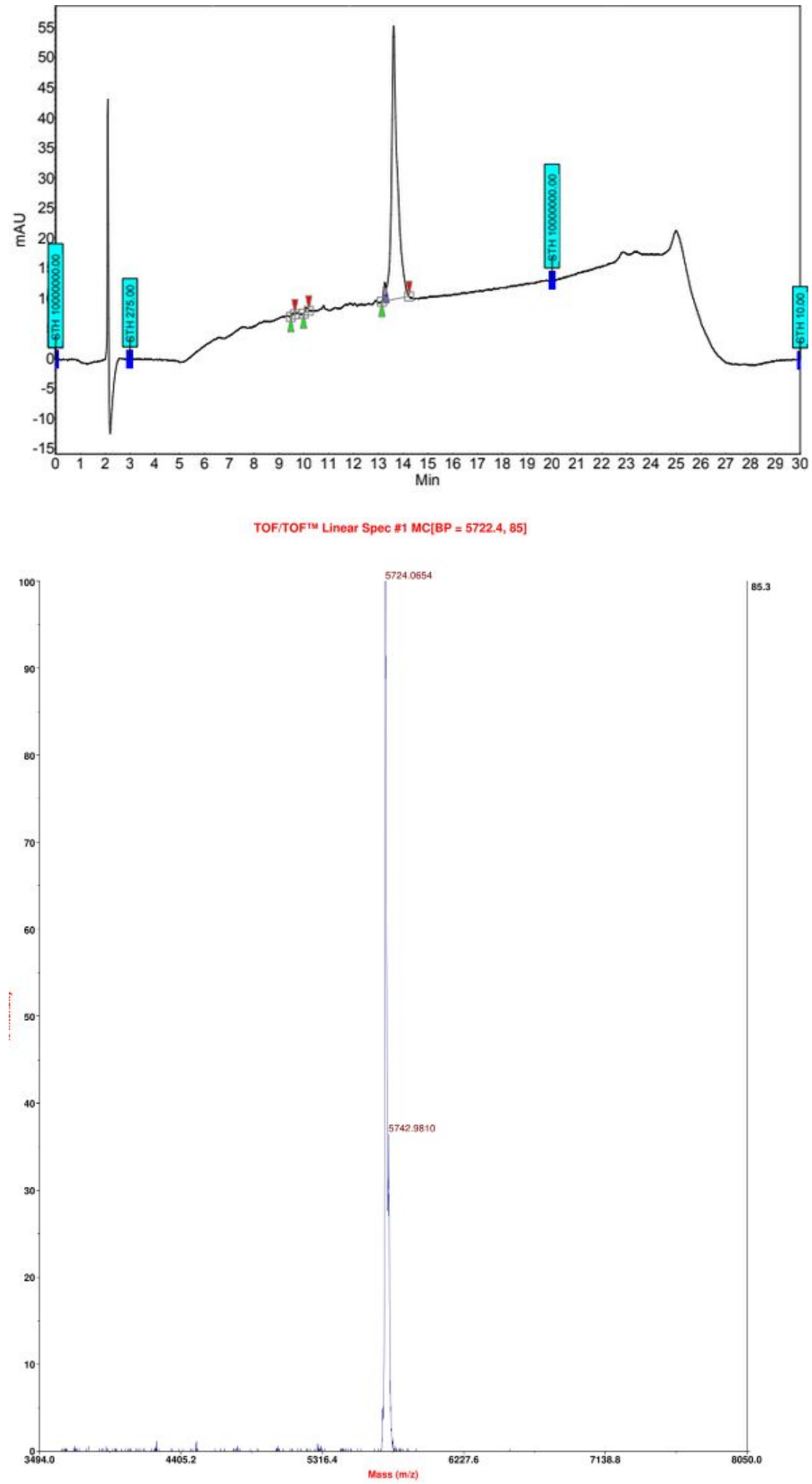

HPLC (5-95\% $20 \mathrm{~min}) \mathrm{RT}=13.61 \mathrm{~min}$

MALDI-TOF calc. $(\mathrm{M}+\mathrm{H})^{+} 5939.68$, obs. $=5722.4$ 


\section{L034 loopoid strand}

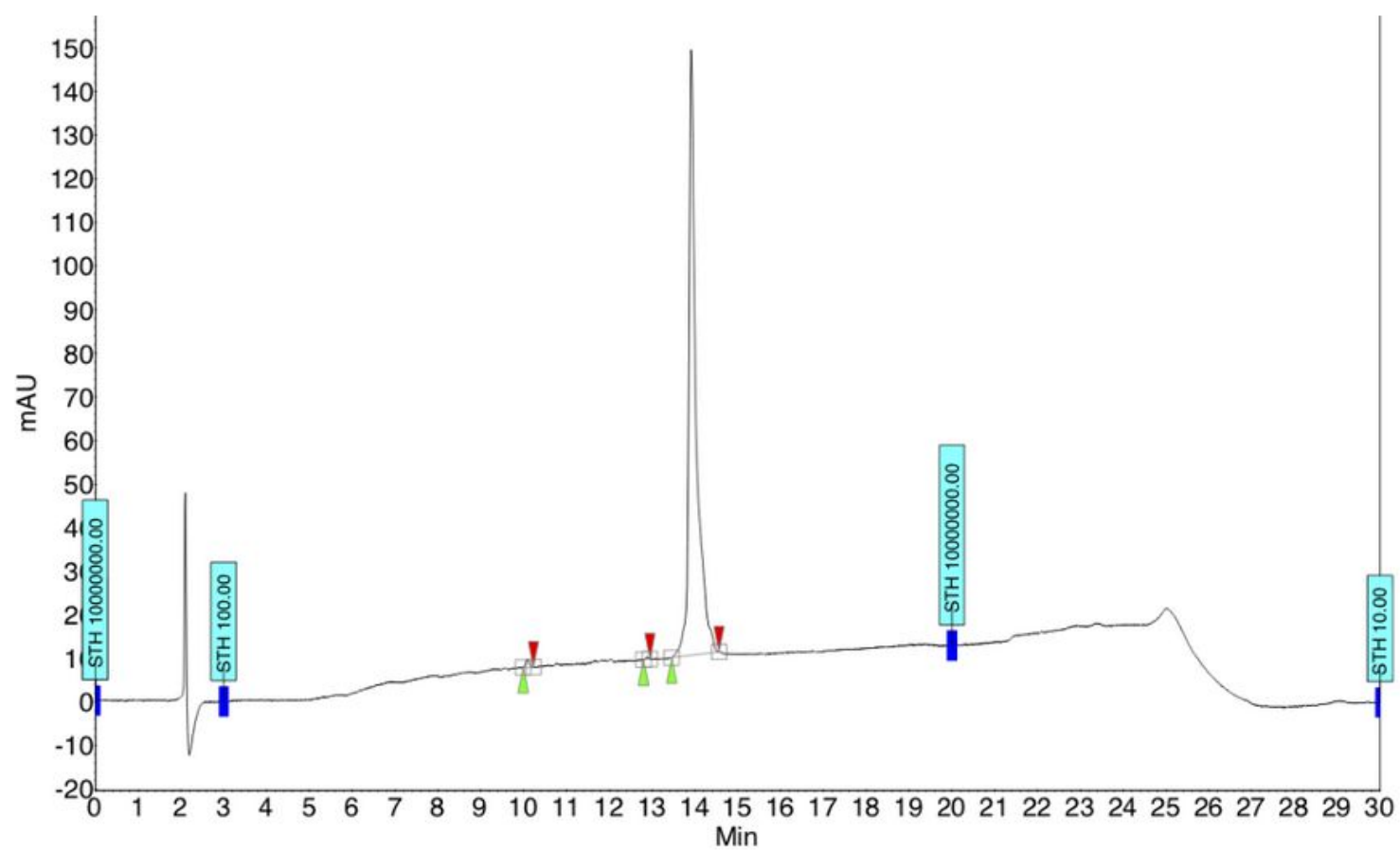

TOF/TOFTM Linear Spec \#1 MC[BP = 5906.7, 25125]

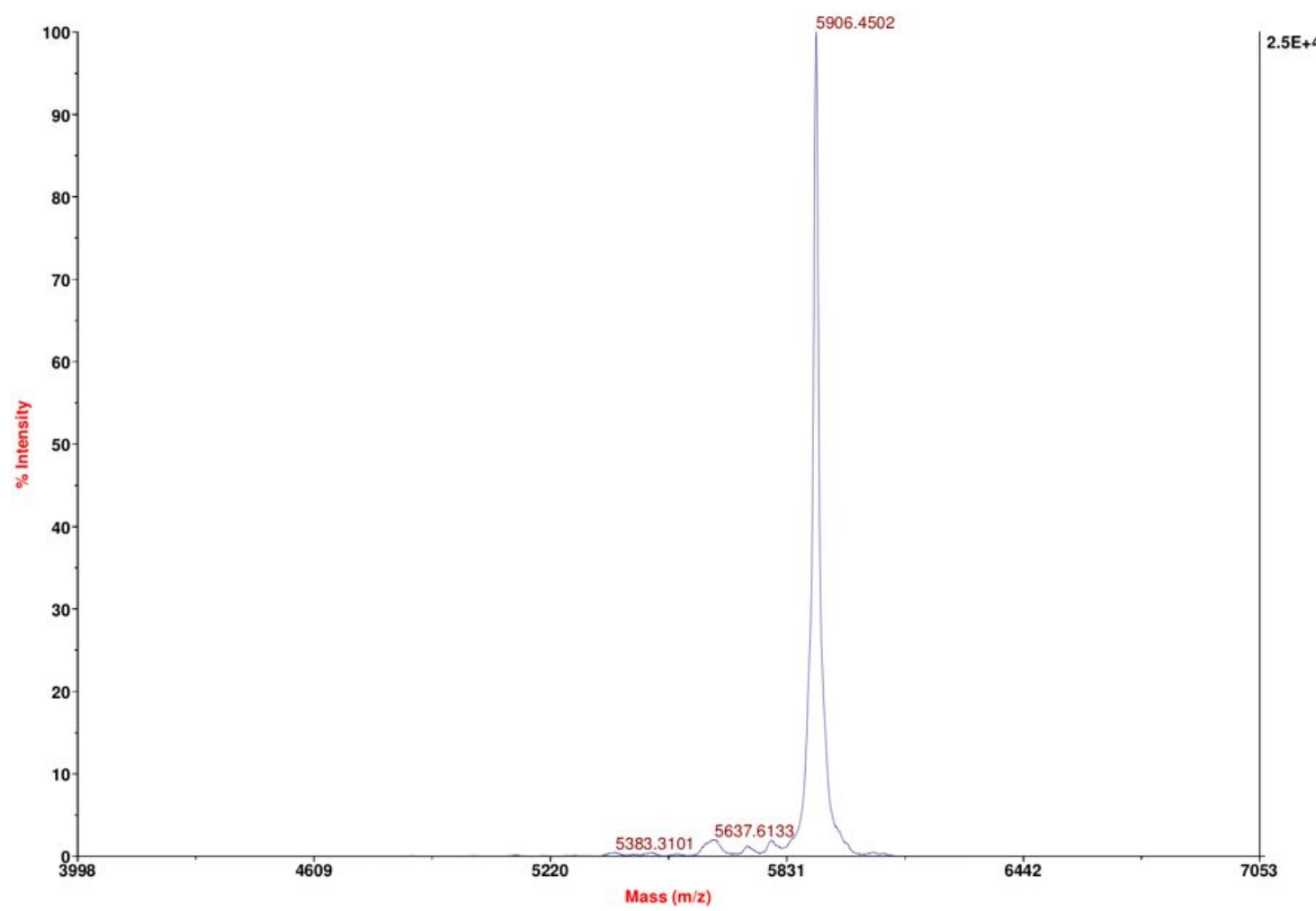

HPLC (5-95\% $20 \mathrm{~min}) \mathrm{RT}=13.91 \mathrm{~min}$

MALDI-TOF calc. $(\mathrm{M}+\mathrm{H})^{+}$5919.85, obs. $=5906.45$ 


\section{TYWWLD Peptide}

2: UV Detector: $214 \mathrm{Nm}$

青 $\quad \stackrel{(1)}{42 \%} T$ (1)

\begin{tabular}{rc|c} 
青 & $42 \%$ \\
毒 & 0.53 & $45 \%$ \\
0.54
\end{tabular}

星 1.0

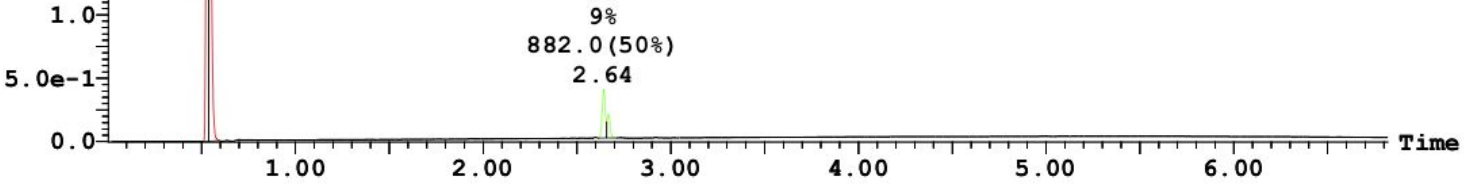

$\begin{array}{rrr}\text { Peak ID } & \text { Time } & \text { Mass Found } \\ 2 & 2.64 & 904.75,441.65,882.61\end{array}$

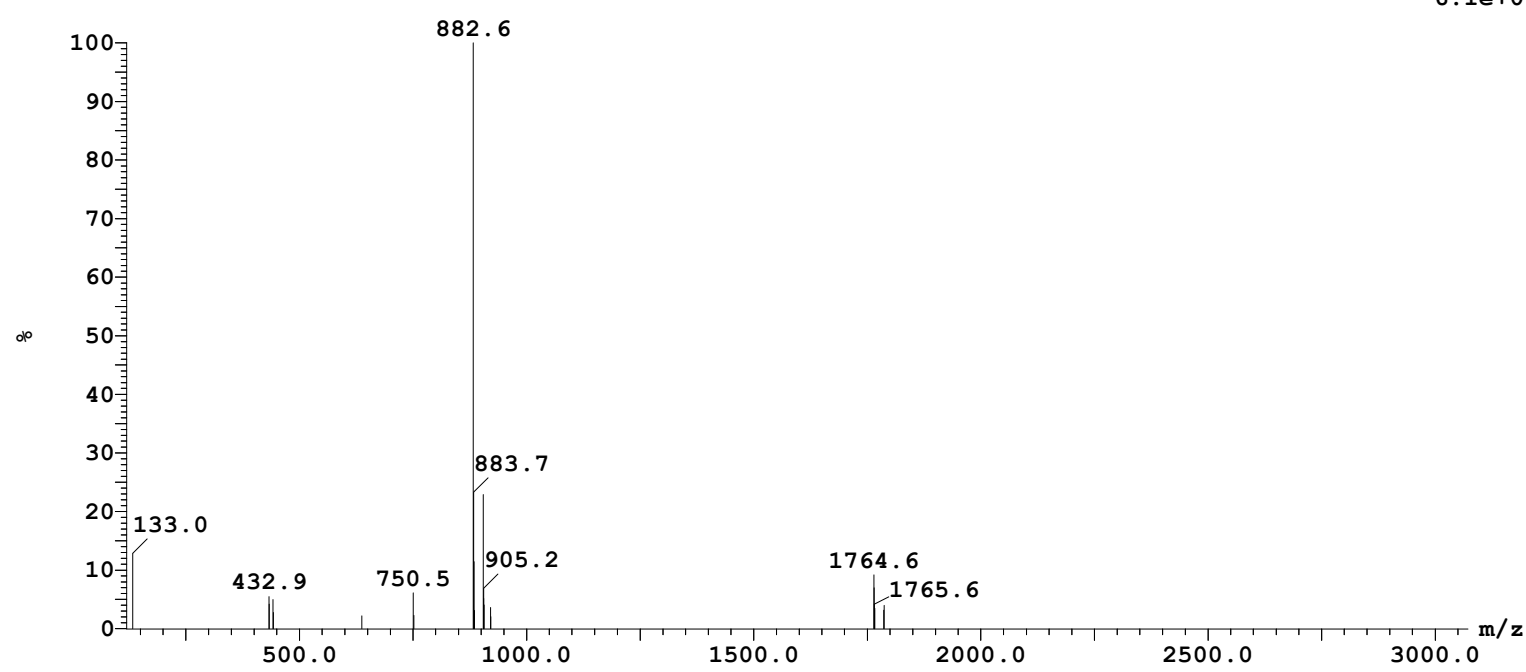

UPLC (5-95\% $6.8 \mathrm{~min}) \mathrm{RT}=2.64 \mathrm{~min}$

ESI-MS calc. $(\mathrm{M}+\mathrm{H})^{+}=882.97$, obs. $=882.6$ 


\section{Table S1. HP pattern, sequence, characterization of loopoids}

+ and - indicates pass and fail, respectively.

n.a. means that loopoid was not applied to synthesis or test for nanosheet formation.

Abbreviations of monomers are as like below.

Nab: Aminobutyl, Nae: Aminoethyl, Nce: Carboxyethyl, Ncm: Carboxymethyl, Namd: Carboxamide, Nhe: Hydroxyethyl, Npp: Propylpyrrolidinone, Nipr: Isopropyl, Ncpr: Cyclopropylmethy, Nbu: N-butyl, Nia: Isoamyl, Ncpe: Cyclopentyl, Ndpe: Diphenylethyl, Nfu: Furfurylamine, Nph: Phenyl, Npe: Phenylethyl, Nbsa: Benzenesulfonamide, Ntyr: Tyramine, Ntrp: Tryptamine, Npip: Piperonyl

\begin{tabular}{|c|c|c|c|c|c|c|c|}
\hline ID & HP pattern & Sequence & M. W. & MALDI (MH+) & Purity & Purified wt. /mg & $\begin{array}{l}\text { Nanosheet } \\
\text { formation }\end{array}$ \\
\hline 1 & \multirow{3}{*}{ HPPHPP } & Ndpe-Ncm-Ncm-Ndpe-Ncm-Ncm & 5916.783 & $1480.17(4 \mathrm{H}+)$ & $95+\%$ & 39 & + \\
\hline 2 & & Ncpe-Nce-Nce-Ncpe-Npp-Npp & 5854.845 & 5847.4263 & $95+\%$ & 18 & + \\
\hline 3 & & Nbn-Ncm-Nab-Nipr-Ncm-Ncm & 5701.576 & 5688.0205 & $95+\%$ & 33 & + \\
\hline 4 & \multirow{3}{*}{ PHHPPP } & Nab-Ntrp-Ntrp-Nab-Nab-Nab & 5895.009 & 5890.1978 & $95+\%$ & 30 & + \\
\hline 5 & & Ncm-Ndpe-Ndpe-Nhe-Nhe-Ncm & 5888.817 & $1472.90(4 \mathrm{H}+)$ & $95+\%$ & 39 & + \\
\hline 6 & & Npp-Nia-Ntrp-Nce-Nce-Npp & 5931.931 & 5926.7642 & $90+\%$ & 25 & + \\
\hline 7 & \multirow{3}{*}{ PHPPHP } & Namd-Ndpe-Namd-Namd-Ndpe-Namd & 5912.847 & 5907.7891 & $95+\%$ & 13 & + \\
\hline 8 & & Nab-Ndpe-Nab-Nab-Ndpe-Namd & 5955.06 & $1489.47(4 \mathrm{H}+)$ & $90+\%$ & 20 & + \\
\hline 9 & & Nce-Nbu-Nae-Nce-Nia-Nce & 5709.64 & 5704.5317 & $95+\%$ & 30 & + \\
\hline 10 & \multirow{3}{*}{ PPHHPP } & Npp-Npp-Ncpr-Ncpr-Npp-Npp & 5933.007 & $5930.852(\mathrm{M}+\mathrm{H})+$ & $95+\%$ & 23 & - \\
\hline 11 & & Nhe-Nae-Ntyr-Ntyr-Nhe-Nae & 5738.685 & 5724.0654 & $95+\%$ & 22 & + \\
\hline 12 & & Npp-Npp-Ntrp-Ncpe-Nab-Nab & 5928.035 & 5922.5142 & $95+\%$ & 26 & + \\
\hline 13 & \multirow{3}{*}{ HHPHPP } & Nipr-Nipr-Nab-Nipr-Nab-Nab & 5663.751 & 5659.6748 & $95+\%$ & 28 & - \\
\hline 14 & & Nipr-Nipr-Nce-Nipr-Nae-Nce & 5637.577 & $95+\%$ & $95+\%$ & 80 & + \\
\hline 15 & & Nia-Nfu-Ncm-Nfu-Npp-Ncm & 5795.689 & $5792.328(\mathrm{M}+\mathrm{H})+$ & $85+\%$ & 18 & + \\
\hline 16 & \multirow{3}{*}{ HHPPHP } & Nbu-Nbu-Npp-Npp-Nbu-Npp & 5867.976 & $5865.745(\mathrm{M}+\mathrm{H})+$ & $95+\%$ & 21 & - \\
\hline 17 & & Nbsa-Nbsa-Npp-Npp-Nbsa-Namd & 6181.208 & 6176.3267 & $95+\%$ & 28 & + \\
\hline 18 & & Nbsa-Ncpr-Namd-Nhe-Ncpr-Nhe & 5760.706 & 5754.2998 & $95+\%$ & 18 & + \\
\hline 19 & \multirow{3}{*}{ HHPPPH } & Ncpr-Ncpr-Npp-Npp-Npp-Ncpr & 5861.928 & $1466.59(4 \mathrm{H}+)$ & $95+\%$ & 43 & + \\
\hline 20 & & Ncpr-Ncpr-Nab-Npp-Nab-Ncpr & 5753.832 & 5748.436 & $95+\%$ & 24 & + \\
\hline 21 & & Ncpe-Nfu-Nab-Nab-Namd-Nfu & 5751.728 & 5739.7236 & $95+\%$ & 28 & + \\
\hline 22 & \multirow{3}{*}{ HPHHPP } & Ntrp-Namd-Ntrp-Ntrp-Namd-Namd & 5924.862 & 5920.917 & $95+\%$ & 11 & + \\
\hline 23 & & Nipr-Nab-Nipr-Nipr-Npp-Nab & 5717.799 & 5705.4771 & $93 \%$ & 15 & + \\
\hline 24 & & Nipr-Nce-Nbu-Nbu-Nae-Nae & 5636.637 & 5634.1123 & $95+\%$ & 16 & + \\
\hline 25 & \multirow{3}{*}{ PHHHPP } & Nae-Ncpe-Ncpe-Ncpe-Nae-Nae & 5657.703 & $943.77(6 \mathrm{H}+)$ & $95+\%$ & 82 & + \\
\hline 26 & & Npp-Npip-Npip-Npip-Npp-Npp & 6102.054 & $1528.08(4 \mathrm{H}+)$ & $95+\%$ & 52 & + \\
\hline 27 & & Namd-Nipr-Nipr-Npip-Npp-Npp & 5849.829 & $1463.22(4 \mathrm{H}+)$ & $90+\%$ & 51 & + \\
\hline 28 & \multirow{3}{*}{ PHHPHP } & Nae-Ncpr-Ncpr-Nae-Ncpr-Nae & 5615.622 & $1404.86(4 \mathrm{H}+)$ & $90+\%$ & 14 & + \\
\hline 29 & & Nhe-Nipr-Nipr-Nce-Nipr-Nce & 5638.561 & $1410.48(4 \mathrm{H}+)$ & $85+\%$ & 18 & + \\
\hline 30 & & Nhe-Nbu-Nia-Nhe-Nia-Npp & 5733.794 & $1433.9(\mathrm{M}+4 \mathrm{H}) 4+$ & $90+\%$ & 22 & + \\
\hline 31 & \multirow{3}{*}{ РPPHHH } & Nce-Nce-Nce-Npip-Npip-Npip & 5942.73 & $1189.34(5 \mathrm{H}+)$ & $95+\%$ & 62 & + \\
\hline 32 & & Nhe-Nhe-Npp-Nia-Nia-Nia & 5747.821 & $5745.711(\mathrm{M}+\mathrm{H})+$ & $90+\%$ & 17 & - \\
\hline 33 & & Npp-Nhe-Nhe-Ndpe-Ndpe-Npe & 6002.068 & $1501.33(4 \mathrm{H}+)$ & $95+\%$ & 28 & + \\
\hline 34 & \multirow{3}{*}{ PHHHHP } & Namd-Ntyr-Ntyr-Ntyr-Ntyr-Namd & 5918.847 & 5904.1196 & $95+\%$ & 36 & + \\
\hline 35 & & Npp-Nbu-Nbu-Nbu-Nbu-Namd & 5730.794 & $1432.9(\mathrm{M}+4 \mathrm{H}) 4+$ & $90+\%$ & 3 & + \\
\hline 36 & & Ncm-Ncpr-Npip-Ncpr-Npip-Npp & 5883.798 & 5879.6567 & $90+\%$ & 13.9 & + \\
\hline
\end{tabular}




\begin{tabular}{|c|c|c|c|c|c|c|c|}
\hline ID & HP pattern & Sequence & M. W. & MALDI (MH+) & Purity & Purified wt. /mg & $\begin{array}{l}\text { Nanosheet } \\
\text { formation }\end{array}$ \\
\hline 37 & \multirow{3}{*}{ HPHPPP } & Ndpe-Ncm-Ncm-Ndpe-Ncm-Ncm & 5908.985 & $1477.85[\mathrm{M}+4 \mathrm{H}]$ & $95 \%$ & 25 & + \\
\hline 38 & & Ncpe-Nce-Nce-Ncpe-Npp-Npp & 6149.205 & $6135.4141[\mathrm{M}+\mathrm{H}]+$ & $95 \%$ & 10 & + \\
\hline 39 & & Nbn-Ncm-Nab-Nipr-Ncm-Ncm & 6005.076 & $1201.9(\mathrm{M}+5 \mathrm{H}) 5+$ & $90+\%$ & 12 & - \\
\hline 40 & \multirow{3}{*}{ HPPPPH } & Nab-Ntrp-Ntrp-Nab-Nab-Nab & 5640.621 & $1411.0(\mathrm{M}+4 \mathrm{H}) 4+$ & $95+\%$ & 18 & n.a. \\
\hline 41 & & Ncm-Ndpe-Ndpe-Nhe-Nhe-Ncm & 5678.626 & $5669.4976[\mathrm{M}+\mathrm{H}]+$ & $95 \%$ & 23 & + \\
\hline 42 & & Npp-Nia-Ntrp-Nce-Nce-Npp & 5759.568 & $5751.0220[\mathrm{M}+\mathrm{H}]+$ & $95+\%$ & 16 & + \\
\hline 43 & \multirow{3}{*}{ PHPHPP } & Namd-Ndpe-Namd-Namd-Ndpe-Namd & 5664.467 & $1416.71[\mathrm{M}+4 \mathrm{H}]$ & $95 \%$ & 22 & + \\
\hline 44 & & Nab-Ndpe-Nab-Nab-Ndpe-Namd & 5780.902 & $1446.0(\mathrm{M}+4 \mathrm{H}) 4+$ & $95+\%$ & 10 & - \\
\hline 45 & & Nce-Nbu-Nae-Nce-Nia-Nce & 5642.604 & $5630.5684[\mathrm{M}+\mathrm{H}]+$ & $90+\%$ & 17 & - \\
\hline 46 & \multirow{3}{*}{ PHPPPH } & Npp-Npp-Ncpr-Ncpr-Npp-Npp & 5604.599 & $1121.76[\mathrm{M}+5 \mathrm{H}]$ & $85 \%$ & 50 & - \\
\hline 47 & & Nhe-Nae-Ntyr-Ntyr-Nhe-Nae & 5756.699 & $1439.9(\mathrm{M}+4 \mathrm{H}) 4+$ & $95 \%$ & 17 & + \\
\hline 48 & & Npp-Npp-Ntrp-Ncpe-Nab-Nab & 5815.727 & $1454.73[\mathrm{M}+4 \mathrm{H}]$ & $70 \%$ & 9 & + \\
\hline 49 & \multirow{3}{*}{ PPPHHP } & Nipr-Nipr-Nab-Nipr-Nab-Nab & 5902.985 & $1476.9(\mathrm{M}+4 \mathrm{H}) 4+$ & n.a. & n.a. & - \\
\hline 50 & & Nipr-Nipr-Nce-Nipr-Nae-Nce & 5698.58 & $1425.34[\mathrm{M}+4 \mathrm{H}]$ & $50 \%$ & 10 & + \\
\hline 51 & & Nia-Nfu-Ncm-Nfu-Npp-Ncm & 5954.965 & $1489.32[\mathrm{M}+4 \mathrm{H}]$ & $95+\%$ & 44 & + \\
\hline 52 & \multirow{3}{*}{ HНHPPP } & Nbu-Nbu-Npp-Npp-Nbu-Npp & 5847.949 & $5841.3472[\mathrm{M}+\mathrm{H}]+$ & $95 \%$ & 6 & + \\
\hline 53 & & Nbsa-Nbsa-Npp-Npp-Nbsa-Namd & 5887.922 & -- & n.a. & n.a. & n.a. \\
\hline 54 & & Nbsa-Ncpr-Namd-Nhe-Ncpr-Nhe & 5778.658 & $5769.9619[\mathrm{M}+\mathrm{H}]+$ & $95+\%$ & 13 & + \\
\hline 55 & \multirow{3}{*}{ HPPHHP } & Ncpr-Ncpr-Npp-Npp-Npp-Ncpr & 5666.571 & $1417.1(\mathrm{M}+4 \mathrm{H}) 4+$ & $90+\%$ & 25 & - \\
\hline 56 & & Ncpr-Ncpr-Nab-Npp-Nab-Ncpr & 5665.682 & $1417.08[\mathrm{M}+4 \mathrm{H}]$ & $95+\%$ & 54 & - \\
\hline 57 & & Ncpe-Nfu-Nab-Nab-Namd-Nfu & 5769.61 & $5764.9434[\mathrm{M}+\mathrm{H}]+$ & $95 \%$ & 8 & + \\
\hline 58 & \multirow{3}{*}{ HPPHPH } & Ntrp-Namd-Ntrp-Ntrp-Namd-Namd & 5967.075 & $5987.4419[\mathrm{M}+\mathrm{Na}]+$ & $90+\%$ & 14 & - \\
\hline 59 & & Nipr-Nab-Nipr-Nipr-Npp-Nab & 5711.795 & $1428.9(\mathrm{M}+4 \mathrm{H}) 4+$ & $95+\% \%$ & 20 & + \\
\hline 60 & & Nipr-Nce-Nbu-Nbu-Nae-Nae & 5810.766 & $5802.439[\mathrm{M}+\mathrm{H}]+$ & $95+\%$ & 19 & + \\
\hline 61 & \multirow{3}{*}{ PHHPPH } & Nae-Ncpe-Ncpe-Ncpe-Nae-Nae & 5904.009 & $1476.57[\mathrm{M}+4 \mathrm{H}]$ & $95+\%$ & 57 & + \\
\hline 62 & & Npp-Npip-Npip-Npip-Npp-Npp & 5833.789 & $1459.0(\mathrm{M}+4 \mathrm{H}) 4+$ & n.a. & n.a. & n.a. \\
\hline 63 & & Namd-Nipr-Nipr-Npip-Npp-Npp & 5744.6 & $5735.6455[\mathrm{M}+\mathrm{H}]+$ & $95+\%$ & 9 & + \\
\hline 64 & \multirow{3}{*}{ РPHНHP } & Nae-Ncpr-Ncpr-Nae-Ncpr-Nae & 5702.604 & $1426.46[\mathrm{M}+4 \mathrm{H}]$ & $95+\%$ & 67 & + \\
\hline 65 & & Nhe-Nipr-Nipr-Nce-Nipr-Nce & 5680.6 & $1424.8(\mathrm{M}+4 \mathrm{H}) 4+$ & $90 \%$ & 16 & n.a. \\
\hline 66 & & Nhe-Nbu-Nia-Nhe-Nia-Npp & 5680.598 & $5672.7271[\mathrm{M}+\mathrm{H}]+$ & $95+\%$ & 29 & - \\
\hline 67 & \multirow{3}{*}{ PPHHPH } & Nce-Nce-Nce-Npip-Npip-Npip & 5882.913 & $5890.3662[\mathrm{M}+\mathrm{Na}]+$ & $95+\%$ & 20 & - \\
\hline 68 & & Nhe-Nhe-Npp-Nia-Nia-Nia & 5900.828 & $1475.52[\mathrm{M}+4 \mathrm{H}]$ & $95+\%$ & 66 & + \\
\hline 69 & & Npp-Nhe-Nhe-Ndpe-Ndpe-Npe & 5955.909 & $1489.9(\mathrm{M}+4 \mathrm{H}) 4+$ & $90 \%$ & 20 & - \\
\hline 70 & \multirow{3}{*}{ РPHРHН } & Namd-Ntyr-Ntyr-Ntyr-Ntyr-Namd & 5768.754 & $1443.0(\mathrm{M}+4 \mathrm{H}) 4+$ & n.a. & n.a. & n.a. \\
\hline 71 & & Npp-Nbu-Nbu-Nbu-Nbu-Namd & 5782.646 & $1446.34[\mathrm{M}+4 \mathrm{H}]$ & --- & 41 & + \\
\hline 72 & & Ncm-Ncpr-Npip-Ncpr-Npip-Npp & 5920.951 & $1480.77[\mathrm{M}+4 \mathrm{H}]$ & --- & 60 & - \\
\hline
\end{tabular}




\begin{tabular}{|c|c|c|c|c|c|c|c|}
\hline ID & HP pattern & Sequence & M. W. & MALDI (MH+) & Purity & Purified wt. /mg & $\begin{array}{c}\text { Nanosheet } \\
\text { formation }\end{array}$ \\
\hline 73 & \multirow{3}{*}{ HHPPPP } & Ndpe-Ncm-Ncm-Ndpe-Ncm-Ncm & 5634.621 & $1409.96[\mathrm{M}+4 \mathrm{H}]$ & $95+\%$ & 80 & - \\
\hline 74 & & Ncpe-Nce-Nce-Ncpe-Npp-Npp & 5756.656 & $1440.41[\mathrm{M}+4 \mathrm{H}]$ & $95+\%$ & 57 & + \\
\hline 75 & & Nbn-Ncm-Nab-Nipr-Ncm-Ncm & 5913.87 & $1479.0[\mathrm{M}+4 \mathrm{H}] 4+$ & $90 \%$ & 30 & n.a. \\
\hline 76 & \multirow{3}{*}{ HPPPHP } & Nab-Ntrp-Ntrp-Nab-Nab-Nab & 5694.673 & $1424.89[\mathrm{M}+4 \mathrm{H}]$ & $95 \%$ & 35 & + \\
\hline 77 & & Ncm-Ndpe-Ndpe-Nhe-Nhe-Ncm & 5628.663 & $1408[\mathrm{M}+4 \mathrm{H}] 4+$ & $95 \%$ & 30 & n.a. \\
\hline 78 & & Npp-Nia-Ntrp-Nce-Nce-Npp & 5672.5532 & n.a. & n.a. & n.a. & n.a. \\
\hline 79 & \multirow{3}{*}{ PPHPHP } & Namd-Ndpe-Namd-Namd-Ndpe-Namd & 5750.871 & $1438.09[\mathrm{M}+4 \mathrm{H}]$ & $90 \%$ & 27 & n.a. \\
\hline 80 & & Nab-Ndpe-Nab-Nab-Ndpe-Namd & 5747.6228 & n.a. & n.a. & n.a. & n.a. \\
\hline 81 & & Nce-Nbu-Nae-Nce-Nia-Nce & 5907.91 & $1478.49(\mathrm{M}+4 \mathrm{H}) 4+$ & $85 \%$ & 5 & + \\
\hline 82 & \multirow{3}{*}{ PPHPPH } & Npp-Npp-Ncpr-Ncpr-Npp-Npp & 6028.9764 & n.a. & n.a. & n.a. & n.a. \\
\hline 83 & & Nhe-Nae-Ntyr-Ntyr-Nhe-Nae & 5974.955 & $5962.3091[\mathrm{M}+\mathrm{H}]+$ & $90 \%$ & 12 & + \\
\hline 84 & & Npp-Npp-Ntrp-Ncpe-Nab-Nab & 5945.1118 & $1487.45(\mathrm{M}+4 \mathrm{H}) 4+$ & $90 \%$ & 25 & + \\
\hline 85 & \multirow{3}{*}{ PPPHPH } & Nipr-Nipr-Nab-Nipr-Nab-Nab & 6057.2445 & $1515.97(\mathrm{M}+4 \mathrm{H}) 4+$ & $90 \%$ & 10 & n.a. \\
\hline 86 & & Nipr-Nipr-Nce-Nipr-Nae-Nce & 5701.576 & $1426.16[\mathrm{M}+4 \mathrm{H}]$ & $95+\%$ & 26 & n.a. \\
\hline 87 & & Nia-Nfu-Ncm-Nfu-Npp-Ncm & 5758.6862 & & & & n.a. \\
\hline 88 & \multirow{3}{*}{ PPPPHH } & Nbu-Nbu-Npp-Npp-Nbu-Npp & 5809.672 & $1453.0(\mathrm{M}+4 \mathrm{H}) 4+$ & $<85 \%$ & 5 & n.a. \\
\hline 89 & & Nbsa-Nbsa-Npp-Npp-Nbsa-Namd & 5854.7284 & n.a. & n.a. & n.a. & n.a. \\
\hline 90 & & Nbsa-Ncpr-Namd-Nhe-Ncpr-Nhe & 5678.582 & $1420.16[\mathrm{M}+4 \mathrm{H}]$ & $90 \%$ & 29 & n.a. \\
\hline 91 & \multirow{3}{*}{ HPHPPH } & Ncpr-Ncpr-Npp-Npp-Npp-Ncpr & 5758.76 & $1440.26[\mathrm{M}+4 \mathrm{H}]$ & $95 \%$ & 46 & n.a. \\
\hline 92 & & Ncpr-Ncpr-Nab-Npp-Nab-Ncpr & 5769.746 & n.a. & n.a. & n.a. & n.a. \\
\hline 93 & & Ncpe-Nfu-Nab-Nab-Namd-Nfu & 5711.6762 & n.a. & n.a. & n.a. & n.a. \\
\hline 94 & \multirow{3}{*}{ HPPPHH } & Ntrp-Namd-Ntrp-Ntrp-Namd-Namd & 6017.004 & n.a. & n.a. & n.a. & n.a. \\
\hline 95 & & Nipr-Nab-Nipr-Nipr-Npp-Nab & 5944.827 & $5931.6548[\mathrm{M}+\mathrm{H}]+$ & $90 \%$ & 14 & + \\
\hline 96 & & Nipr-Nce-Nbu-Nbu-Nae-Nae & 5693.648 & $5684.2407[\mathrm{M}+\mathrm{H}]+$ & $92 \%$ & 14 & + \\
\hline 97 & \multirow{3}{*}{ PHPHHP } & Nae-Ncpe-Ncpe-Ncpe-Nae-Nae & 5679.746 & $1420.76[\mathrm{M}+4 \mathrm{H}]$ & $95 \%$ & 43 & n.a. \\
\hline 98 & & Npp-Npip-Npip-Npip-Npp-Npp & 5699.738 & $1425.9[\mathrm{M}+4 \mathrm{H}]+$ & $95 \%$ & 12 & n.a. \\
\hline 99 & & Namd-Nipr-Nipr-Npip-Npp-Npp & 5792.681 & n.a. & n.a. & n.a. & n.a. \\
\hline 100 & \multirow{3}{*}{ PHPPHH } & Nae-Ncpr-Ncpr-Nae-Ncpr-Nae & 5629.605 & $1408.16[\mathrm{M}+4 \mathrm{H}]$ & $80 \%$ & 9 & n.a. \\
\hline 101 & & Nhe-Nipr-Nipr-Nce-Nipr-Nce & 5907.911 & $1477.9[\mathrm{M}+4 \mathrm{H}] 4+$ & $87-89 \%$ & 10 & n.a. \\
\hline 102 & & Nhe-Nbu-Nia-Nhe-Nia-Npp & 5717.707 & $1430.29[\mathrm{M}+4 \mathrm{H}]$ & $80 \%$ & 7 & n.a. \\
\hline 103 & \multirow{3}{*}{ HPHPHP } & Nce-Nce-Nce-Npip-Npip-Npip & 5645.5708 & $5634.0264[\mathrm{M}+\mathrm{H}]+$ & $85 \%$ & 9 & n.a. \\
\hline 104 & & Nhe-Nhe-Npp-Nia-Nia-Nia & 5853.86 & $5845.3564[\mathrm{M}+\mathrm{H}]+$ & $94 \%$ & 14 & + \\
\hline 105 & & Npp-Nhe-Nhe-Ndpe-Ndpe-Npe & 5775.884 & n.a. & n.a. & n.a. & + \\
\hline 106 & \multirow{3}{*}{ HНPPHН } & Namd-Ntyr-Ntyr-Ntyr-Ntyr-Namd & 5682.753 & $1421.44[\mathrm{M}+4 \mathrm{H}]$ & $95 \%$ & 45 & - \\
\hline 107 & & Npp-Nbu-Nbu-Nbu-Nbu-Namd & 5993.149 & $1499.15[\mathrm{M}+4 \mathrm{H}]$ & $95 \%$ & 49 & - \\
\hline 108 & & Ncm-Ncpr-Npip-Ncpr-Npip-Npp & 5724.839 & $1431.9[\mathrm{M}+4 \mathrm{H}] 4+$ & $90 \%$ & 24 & - \\
\hline
\end{tabular}




\begin{tabular}{|c|c|c|c|c|c|c|}
\hline ID & HP pattern & Sequence & M. W. & MALDI (MH+) & Purity & Purified wt. /mg \\
\hline 109 & \multirow{3}{*}{ HPPHPP } & Nipr-Nhe-Nhe-Ncpe-Nhe-Nhe & 5610.551 & $1403.88[\mathrm{M}+4 \mathrm{H}]$ & $95 \%$ & 2 \\
\hline 110 & & Nph-Namd-Namd-Ncpr-Ncm-Ncm & 5684.51 & n.a. & n.a. & n.a. \\
\hline 111 & & Nipr-Nmp-Nmp-Ntrp-Nab-Nce & 5866.819 & $5855.0308[\mathrm{M}+\mathrm{H}]+$ & $93 \%$ & 15 \\
\hline 112 & \multirow{3}{*}{ PHHPPP } & Npp-Ncpe-Ntyr-Npp-Npp-Npp & 6013.093 & $7155.413[\mathrm{M}+\mathrm{H}]+$ & $95+\%$ & 24 \\
\hline 113 & & Npp-Nbsa-Nia-Nae-Nae-Npp & 5913.98 & $1183.9[\mathrm{M}+4 \mathrm{H}] 4+$ & $95 \%$ & 17 \\
\hline 114 & & Nab-Nph-Nfu-Nmp-Npp-Nab & 5854.852 & $5843.3057[\mathrm{M}+\mathrm{H}]+$ & $92 \%$ & 28 \\
\hline 115 & \multirow{3}{*}{ PHPPHP } & Ncm-Nia-Ncm-Ncm-Nipr-Ncm & 5668.499 & $1418.06[\mathrm{M}+4 \mathrm{H}]$ & $90 \%$ & 26 \\
\hline 116 & & Namd-Ncpr-Namd-Nae-Ncpe-Nae & 5646.5616 & n.a. & n.a. & n.a. \\
\hline 117 & & Nae-Npe-Nae-Ncm-Nia-Nme & 5700.68 & $1426.24[\mathrm{M}+4 \mathrm{H}]$ & $90 \%$ & 42 \\
\hline 118 & \multirow{3}{*}{ PPHHPP } & Nhe-Nhe-Ndpe-Ncpr-Nhe-Nhe & 5734.693 & $1434.49[\mathrm{M}+4 \mathrm{H}]$ & $95 \%$ & 5 \\
\hline 119 & & Nce-Nce-Nipr-Nph-Namd-Namd & 5700.55 & n.a. & n.a. & n.a. \\
\hline 120 & & Npp-Nab-Ncpr-Ntyr-Nce-Npp & 5891.91 & $5877.7261[\mathrm{M}+\mathrm{H}]+$ & $95 \%$ & 24 \\
\hline 121 & \multirow{3}{*}{ HHPHPP } & Ntyr-Nipr-Nme-Nipr-Nme-Nme & 5702692 & $5695.7388[\mathrm{M}+\mathrm{H}]+$ & $95 \%$ & 28 \\
\hline 122 & & Nia-Ntyr-Nae-Ntyr-Nce-Nce & 5821.771 & $5819.2891[\mathrm{M}+\mathrm{H}]+$ & $95 \%$ & 36 \\
\hline 123 & & Ndpe-Ndpe-Nce-Ncpr-Namd-Nae & 5910.92 & n.a. & n.a. & n.a. \\
\hline 124 & \multirow{3}{*}{ HHPPHP } & Ntrp-Ncpr-Nme-Nme-Ncpr-Nme & 5749.752 & n.a. & n.a. & n.a. \\
\hline 125 & & Nbu-Nbu-Namd-Ncm-Ndpe-Ncm & 5789.729 & $1448.44[\mathrm{M}+4 \mathrm{H}]$ & $90 \%$ & 9 \\
\hline 126 & & Nspe-Nspe-Npp-Nce-Nbu-Nab & 5842.93 & n.a. & n.a. & n.a. \\
\hline 127 & \multirow{3}{*}{ HHPPPH } & Npip-Nbu-Nce-Nce-Nce-Npip & 5864.704 & $1467.12[\mathrm{M}+4 \mathrm{H}]$ & $90 \%$ & 42 \\
\hline 128 & & Ndpe-Ncpr-Nab-Ncm-Ncm-Ndpe & 5925.926 & $1482.35[\mathrm{M}+4]$ & $90 \%$ & 18 \\
\hline 129 & & Nipr-Nspe-Nme-Nab-Nmp-Nipr & 5747.78 & $5739.9175[\mathrm{M}+\mathrm{H}]+$ & $95 \%$ & 35 \\
\hline & \multirow{4}{*}{ HPHHPP } & & & & & \\
\hline 130 & & Ntrp-Nme-Ntrp-Nfu-Nme-Nme & 5864.8309 & n.a. & n.a. & n.a. \\
\hline 131 & & Ndpe-Nae-Nipr-Nipr-Nae-Npp & 5799.86 & $1450.77[\mathrm{M}+4 \mathrm{H}]$ & $95 \%$ & 26 \\
\hline 132 & & Nfu-Ncm-Nfu-Nspe-Nhe-Nhe & 5762.66 & n.a. & n.a. & n.a. \\
\hline 133 & \multirow{3}{*}{ PHHHPP } & Ncm-Nbn-Ncpe-Nbn-Ncm-Ncm & 5746.616 & $1437.64[\mathrm{M}+4 \mathrm{H}]$ & $90 \%$ & 16 \\
\hline 134 & & Namd-Ncpr-Ncpr-Nph-Nab-Nab & 5707.72 & n.a. & n.a. & n.a. \\
\hline 135 & & Nab-Ncpr-Ncpr-Nbn-Ncm-Nhe & 5695.66 & $1424.66[\mathrm{M}+4 \mathrm{H}]$ & $90 \%$ & 13 \\
\hline 136 & \multirow{3}{*}{ PHHPHP } & Npp-Nspe-Nbu-Npp-Nspe-Npp & 5964.06 & n.a. & $90 \%$ & 32 \\
\hline 137 & & Npp-Nipr-Nipr-Nae-Ndpe-Nae & 5799.86 & $1450.92[\mathrm{M}+4 \mathrm{H}]$ & $90 \%$ & 20 \\
\hline 138 & & Nmp-Nia-Nia-Namd-Ndpe-Nab & 5879.914 & $5868.7896[\mathrm{M}+\mathrm{H}]+$ & $75 \%$ & 9 \\
\hline 139 & \multirow{3}{*}{ РPPHHH } & Nce-Nce-Nce-Ncpr-Nbsa-Nbsa & 5960.87 & n.a. & n.a. & n.a. \\
\hline 140 & & Nae-Nfu-Nae-Nipr-Ncpe-Nipr & 5642.6134 & $5632.5654[\mathrm{M}+\mathrm{H}]+$ & $86 \%$ & 17 \\
\hline 141 & & Nae-Nme-Nce-Nipr-Nipr-Nia & 5651.648 & $1413.93[\mathrm{M}+4 \mathrm{H}]$ & $90 \%$ & 48 \\
\hline & & & & & & \\
\hline 142 & \multirow{3}{*}{ РHHHHP } & Nab-Ncpr-Nph-Ncpr-Ncpr-Nab & 5704.76 & 5904.1196 & $95+\%$ & 45 \\
\hline 143 & & Nmp-Npip-Nipr-Npip-Nipr-Namd & 5840.7046 & $1432.9(\mathrm{M}+4 \mathrm{H}) 4+$ & $90+\%$ & 49 \\
\hline 144 & & Nae-Ncpe-Ncpr-Ncpr-Nce-Namd & 5672.63 & 5879.6567 & $90+\%$ & 24 \\
\hline
\end{tabular}




\begin{tabular}{|c|c|c|c|c|c|c|}
\hline ID & HP pattern & Sequence & M. W. & MALDI (MH+) & Purity & Purified wt. /mg \\
\hline 145 & \multirow{3}{*}{ HPHPPP } & Ncpr-Nce-Ncpr-Nce-Npp-Nce & 5773.683 & $1444.09[\mathrm{M}+4 \mathrm{H}]$ & $95 \%$ & 83 \\
\hline 146 & & Nipr-Namd-Nfu-Nab-Nab-Namd & 5702.66 & n.a. & n.a. & n.a. \\
\hline 147 & & Nbn-Namd-Nbu-Nae-Nae-Ncm & 5671.6 & n.a. & n.a. & n.a. \\
\hline 148 & \multirow{3}{*}{ HPPPPH } & Nph-Namd-Namd-Nfu-Namd-Nph & 57275486 & na & na & na \\
\hline 149 & & Nfu-Nhe-Nme-Nhe-Nme-Ntyr & 5728.6317 & $5721.6099[\mathrm{M}+\mathrm{H}]+$ & $75 \%$ & 6 \\
\hline 150 & & Npip-Nmp-Nab-Ncm-Ncm-Nia & 5822.6868 & n.a. & n.a. & n.a. \\
\hline 151 & \multirow{3}{*}{ PHPHPP } & Npp-Nipr-Nme-Nipr-Npp-Npp & 5841.894 & $1461.64[\mathrm{M}+4 \mathrm{H}]$ & $95 \%$ & 56 \\
\hline 152 & & Nmp-Nph-Nae-Nbu-Nae-Nmp & 5756.6941 & $5747.9019[\mathrm{M}+\mathrm{H}]+$ & $95 \%$ & 8 \\
\hline 153 & & Namd-Ncpr-Nce-Nia-Nab-Nab & 5719.6962 & n.a. & n.a. & n.a. \\
\hline 154 & \multirow{3}{*}{ PHPPPH } & Ncm-Ndpe-Nhe-Nhe-Ncm-Ndpe & 5888.82 & n.a. & n.a. & n.a. \\
\hline 155 & & Npp-Ndpe-Nab-Nab-Npp-Nph & 5973.075 & n.a. & n.a. & n.a. \\
\hline 156 & & Nme-Ndpe-Npp-Npp-Nae-Ncpr & 5909.972 & $1478.60[\mathrm{M}+4 \mathrm{H}]$ & $90 \%$ & 97 \\
\hline 157 & \multirow{3}{*}{ PPPHHP } & Nme-Nae-Nme-Ndpe-Ndpe-Nae & 5886.937 & $1472.45[\mathrm{M}+4 \mathrm{H}]$ & $95 \%$ & 64 \\
\hline 158 & & Nce-Nab-Nce-Nia-Ncpr-Nce & 5735.678 & $1434.71[\mathrm{M}+4 \mathrm{H}]$ & $95 \%$ & 55 \\
\hline 159 & & Npp-Npp-Nmp-Ntrp-Nipr-Namd & 5923.8836 & n.a. & n.a. & n.a. \\
\hline 160 & \multirow{3}{*}{ HHHPPP } & Nipr-Nipr-Nipr-Ncm-Nhe-Ncm & 5610.507 & $1403.58[\mathrm{M}+4 \mathrm{H}]$ & $95 \%$ & 33 \\
\hline 161 & & Nfu-Nipr-Nipr-Nab-Nab-Nce & 5702.696 & $1426.69[\mathrm{M}+4 \mathrm{H}]$ & $95 \%$ & 96 \\
\hline 162 & & Ncpr-Npip-Ncpr-Namd-Npp-Nhe & 5792.7038 & n.a. & n.a. & n.a. \\
\hline 163 & \multirow{3}{*}{ HPPHHP } & Nbu-Ncm-Nhe-Nbu-Nbu-Ncm & 5652.588 & $1413.93[\mathrm{M}+4 \mathrm{H}]$ & $90 \%$ & 60 \\
\hline 164 & & Nipr-Nae-Namd-Nbu-Nipr-Nae & 5607.5682 & n.a. & n.a. & n.a. \\
\hline 165 & & Nfu-Nme-Nab-Nspe-Nfu-Nhe & 5761.72 & n.a. & n.a. & n.a. \\
\hline 166 & \multirow{3}{*}{ HPPHPH } & Nbsa-Namd-Ncm-Nbsa-Namd-Nbsa & 6045.9618 & $6036.9316[\mathrm{M}+\mathrm{H}]+$ & $95 \%$ & 15 \\
\hline 167 & & Npip-Ncm-Nab-Ncpr-Nab-Ncpr & 5766.739 & $1442.66[\mathrm{M}+4 \mathrm{H}]$ & $95 \%$ & 63 \\
\hline 168 & & Nbu-Nae-Npp-Ndpe-Ncm-Nbu & 5842.881 & $1462.02[\mathrm{M}+4 \mathrm{H}]$ & $95 \%$ & 51 \\
\hline & & & & & & \\
\hline 169 & \multirow{3}{*}{ PHHPPH } & Npp-Nia-Nia-Nmp-Nmp-Nia & 5873.9256 & $5865.2129[\mathrm{M}+\mathrm{H}]+$ & $90 \%$ & 22 \\
\hline 170 & & Nae-Ncpr-Nspe-Npp-Npp-Nspe & 5879.95 & $1470.9[\mathrm{M}+4 \mathrm{H}] 4+$ & $95 \%$ & 34 \\
\hline 171 & & Nae-Nspe-Nspe-Ncm-Nhe-Nipr & 5719.68 & n.a. & n.a. & n.a. \\
\hline 172 & \multirow{3}{*}{ PPHHHP } & Nhe-Nce-Ncpe-Ncpe-Ncpe-Nce & 5716.675 & $1430.29[\mathrm{M}+4 \mathrm{H}]$ & $90 \%$ & 35 \\
\hline 173 & & Nhe-Nme-Nph-Npe-Npe-Nme & 5768.75 & n.a. & n.a. & n.a. \\
\hline 174 & & Nme-Namd-Nia-Nbsa-Ncpe-Nce & 5832.81 & n.a. & n.a. & n.a. \\
\hline 175 & \multirow{3}{*}{ PPHHPH } & Nmp-Nce-Ncpr-Ncpr-Nce-Ncpr & 5737.6246 & n.a. & n.a. & n.a. \\
\hline 176 & & Nme-Ncm-Ntrp-Ncpr-Nme-Ntrp & 5838.793 & n.a. & n.a. & n.a. \\
\hline 177 & & Nmp-Nab-Ntyr-Nipr-Nce-Nipr & 5778.738 & $5765.2773[\mathrm{M}+\mathrm{H}]+$ & $95 \%$ & 27 \\
\hline 178 & \multirow{3}{*}{ PPHPHH } & Nab-Ncm-Ncpr-Nab-Ncpr-Ncpr & 5686.697 & $1422.71[\mathrm{M}+4 \mathrm{H}]$ & $95 \%$ & 38 \\
\hline 179 & & Nce-Nab-Ntyr-Nab-Nipr-Nipr & 5742.7484 & $5730.5815[\mathrm{M}+\mathrm{H}]+$ & $95 \%$ & 32 \\
\hline 180 & & Nae-Ncm-Nbsa-Namd-Nbsa-Ndpe & 6029 & n.a. & n.a. & n.a. \\
\hline
\end{tabular}




\begin{tabular}{|c|c|c|c|c|c|c|}
\hline ID & HP pattern & Sequence & M. W. & MALDI (MH+) & Purity & Purified wt. /mg \\
\hline 181 & \multirow{3}{*}{ HHPPPP } & Ncpe-Ncpe-Nae-Nhe-Nae-Nhe & 5634.621 & $1409.81[\mathrm{M}+4 \mathrm{H}]$ & $90 \%$ & 45 \\
\hline 182 & & Nbsa-Nbu-Npp-Npp-Nce-Nce & 6218.8909 & n.a. & $90 \%$ & 35 \\
\hline 183 & & Ndpe-Nia-Nme-Nhe-Nhe-Nhe & 5764.763 & $1442.51[\mathrm{M}+4 \mathrm{H}]$ & $95 \%$ & 22 \\
\hline 184 & \multirow{3}{*}{ HPPPHP } & Nipr-Nce-Nce-Nae-Nipr-Nce & 5667.559 & $1417.76[\mathrm{M}+4 \mathrm{H}]$ & $95 \%$ & 67 \\
\hline 185 & & Ndpe-Ncm-Ncm-Ncm-Ncpr-Nab & 5803.712 & $1451.89[\mathrm{M}+4 \mathrm{H}]$ & $90 \%$ & 60 \\
\hline 186 & & Nph-Ncm-Ncm-Npp-Ncpr-Ncm & 5753.5982 & n.a. & n.a. & n.a. \\
\hline 187 & \multirow{3}{*}{ PPHPHP } & Namd-Nce-Ndpe-Nce-Ndpe-Nce & 5957.8694 & n.a. & n.a. & n.a. \\
\hline 188 & & Nae-Ncm-Nbsa-Ncm-Nipr-Nae & 5751.6501 & n.a. & n.a. & n.a. \\
\hline 189 & & Nab-Nhe-Nia-Nab-Ncpr-Nhe & 5678.718 & $1420.76[\mathrm{M}+4 \mathrm{H}]$ & $90 \%$ & 55 \\
\hline 190 & \multirow{3}{*}{ PPHPPH } & Ncm-Ncm-Nia-Ncm-Nme-Nia & 5696.597 & $1425.11[\mathrm{M}+4 \mathrm{H}]$ & $90 \%$ & 28 \\
\hline 191 & & Npp-Nae-Nbu-Nae-Npp-Nipr & 5758.808 & $1440.49[\mathrm{M}+4 \mathrm{H}]$ & $90 \%$ & 43 \\
\hline 192 & & Nhe-Nhe-Ndpe-Nab-Nhe-Nfu & 5787.757 & $1447.87[\mathrm{M}+4 \mathrm{H}]$ & $90 \%$ & 17 \\
\hline 193 & \multirow{3}{*}{ PPPHPH } & Namd-Nae-Namd-Ntrp-Nae-Ntrp & 5810.7276 & n.a. & n.a. & n.a. \\
\hline 194 & & Namd-Namd-Nae-Ntyr-Nae-Ndpe & 5824.782 & $5814.7749[\mathrm{M}+\mathrm{H}]+$ & $85 \%$ & 26 \\
\hline 195 & & Nme-Nme-Nme-Nph-Nae-Ncpe & 5685.67 & n.a. & n.a. & n.a. \\
\hline 196 & \multirow{3}{*}{ PPPPHH } & Npp-Npp-Nme-Nme-Nfu-Nfu & 5850.813 & $1463.74[\mathrm{M}+4 \mathrm{H}]$ & $95 \%$ & 39 \\
\hline 197 & & Nae-Nce-Nce-Nce-Nbu-Ntyr & 5759.6458 & $5751.9385[\mathrm{M}+\mathrm{H}]+$ & $95 \%$ & 34 \\
\hline 198 & & Nme-Nae-Nae-Nme-Ncpr-Ntrp & 5723.705 & n.a. & n.a. & n.a. \\
\hline 199 & \multirow{3}{*}{ HPHPPH } & Nipr-Nhe-Nipr-Nhe-Nab-Nipr & 5609.611 & $1403.58[\mathrm{M}+4 \mathrm{H}]$ & $95 \%$ & 58 \\
\hline 200 & & Npip-Npp-Npip-Npp-Nab-Nspe & 6018.0118 & n.a. & n.a. & n.a. \\
\hline 201 & & Nfu-Nhe-Nbn-Nce-Nce-Nspe & 5786.681 & n.a. & n.a. & n.a. \\
\hline 202 & \multirow{3}{*}{ HPPPHH } & Ncpr-Namd-Namd-Nme-Ncpr-Ncpr & 5658.5871 & n.a. & n.a. & n.a. \\
\hline 203 & & Ndpe-Nme-Ncm-Nme-Nia-Nia & 5818.855 & $1455.72[\mathrm{M}+4 \mathrm{H}]$ & $95 \%$ & 78 \\
\hline 204 & & Ncpe-Namd-Nce-Nce-Npe-Nipr & 5739.658 & n.a. & n.a. & n.a. \\
\hline 205 & \multirow{3}{*}{ PHPHHP } & Npp-Ncpr-Npp-Ncpr-Ncpr-Nmp & 5843.856 & $5829.4287[\mathrm{M}+\mathrm{H}]+$ & $92 \%$ & 23 \\
\hline 206 & & Npp-Nph-Npp-Ncpr-Ncpr-Nme & 5816.84 & n.a. & n.a. & n.a. \\
\hline 207 & & Nab-Ntyr-Nme-Ncpe-Nipr-Nme & 5741.7606 & $5731.7388[\mathrm{M}+\mathrm{H}]+$ & $92 \%$ & 38 \\
\hline 208 & \multirow{3}{*}{ PHPPHH } & Nce-Ncpr-Namd-Namd-Ncpr-Ncpr & 5672.58 & n.a. & n.a. & n.a. \\
\hline 209 & & Nae-Nipr-Namd-Namd-Npip-Nipr & 5699.5788 & n.a. & n.a. & n.a. \\
\hline 210 & & Nae-Nbn-Nmp-Nmp-Nspe-Nbu & 5831.804 & $5824.9771[\mathrm{M}+\mathrm{H}]+$ & $90 \%$ & 28 \\
\hline 211 & \multirow{3}{*}{ HPHPHP } & Ncpe-Nab-Ncpe-Nmp-Ncpe-Nab & 5777.8218 & n.a. & n.a. & n.a. \\
\hline 212 & & Nspe-Nab-Ntrp-Nce-Nspe-Nab & 5889.9274 & n.a. & n.a. & n.a. \\
\hline 213 & & Nipr-Nab-Ntrp-Nab-Nph-Npp & 5852.9097 & $5845.0396[\mathrm{M}+\mathrm{H}]+$ & $70 \%$ & 16 \\
\hline 214 & \multirow{3}{*}{ HНPPHH } & Nipr-Nipr-Namd-Namd-Nipr-Nipr & 5606.5372 & n.a. & n.a. & n.a. \\
\hline 215 & & Nfu-Nspe-Ncm-Nab-Npe-Npe & 5845.84 & n.a. & n.a. & n.a. \\
\hline 216 & & Ncpr-Npip-Nae-Ncm-Ncpr-Ndpe & 5847.812 & $1463.29[\mathrm{M}+4 \mathrm{H}]$ & $95 \%$ & 68 \\
\hline
\end{tabular}




\begin{tabular}{|c|c|c|c|c|c|c|}
\hline ID & HP pattern & Sequence & M. W. & MALDI (MH+) & Purity & Purified wt. /mg \\
\hline 217 & \multirow{3}{*}{ HPPPPH } & Ndpe-Namd-Namd-Nme-Nme-Ndpe & 5910.94 & n.a. & n.a. & n.a. \\
\hline 218 & & Ncpr-Nce-Npp-Npp-Nce-Ntrp & 5917.904 & $5905.8164[\mathrm{M}+\mathrm{H}]+$ & $60 \%$ & 13 \\
\hline 219 & & Nia-Npp-Nce-Nce-Nce-Ndpe & 5915.884 & $1479.87[\mathrm{M}+4 \mathrm{H}]$ & $95 \%$ & 106 \\
\hline 220 & \multirow{3}{*}{ PHPPHP } & Nab-Ndpe-Npp-Nab-Ndpe-Npp & 6077.227 & $1519.78[\mathrm{M}+4 \mathrm{H}]$ & $90 \%$ & 118 \\
\hline 221 & & Npp-Nia-Npp-Nae-Nipr-Nae & 5772.835 & $1444.09[\mathrm{M}+4 \mathrm{H}]$ & $95 \%$ & 88 \\
\hline 222 & & Nhe-Ndpe-Nab-Nhe-Nbu-Nab & 5790.849 & $1448.52[\mathrm{M}+4 \mathrm{H}]$ & $95 \%$ & 110 \\
\hline 223 & \multirow{3}{*}{ PPHPPH } & Ncm-Nme-Nfu-Nme-Ncm-Nfu & 5716.543 & $1430.21[\mathrm{M}+4 \mathrm{H}]$ & $90 \%$ & 21 \\
\hline 224 & & Nfu-Nhe-Ncpr-Nhe-Nhe-Npip & 5724.5826 & n.a. & n.a. & n.a. \\
\hline 225 & & Nce-Nme-Nph-Nme-Nce-Nspe & 5764.68 & n.a. & n.a. & n.a. \\
\hline 226 & \multirow{3}{*}{ HНHPPP } & Nipr-Nipr-Nipr-Nmp-Nme-Nme & 5673.6244 & n.a. & n.a. & n.a. \\
\hline 227 & & Ncpr-Ncpr-Ntrp-Nhe-Nae-Nae & 5705.6898 & n.a. & n.a. & n.a. \\
\hline 228 & & Nspe-Nia-Npe-Namd-Ncm-Namd & 5774.72 & n.a. & n.a. & n.a. \\
\hline 229 & \multirow{3}{*}{ HHPPHP } & Nia-Nia-Ncm-Nab-Nia-Ncm & 5721.739 & $1431.64[\mathrm{M}+4 \mathrm{H}]$ & $95 \%$ & 16 \\
\hline 230 & & Nipr-Ntrp-Nae-Nhe-Nipr-Nae & 5681.6678 & n.a. & n.a. & n.a. \\
\hline 231 & & Nspe-Ncpe-Nhe-Npp-Nipr-Npp & 5832.89 & n.a. & n.a. & n.a. \\
\hline 232 & \multirow{3}{*}{ HPHHPP } & Nipr-Namd-Nipr-Nipr-Nme-Namd & 5622.57 & n.a. & n.a. & n.a. \\
\hline 233 & & Npip-Ncm-Ncpr-Ncpr-Nab-Ncm & 5753.652 & $1439.59[\mathrm{M}+4 \mathrm{H}]$ & $95 \%$ & 70 \\
\hline 234 & & Nph-Nhe-Ncpr-Nbu-Ncm-Ncm & 5638.52 & n.a. & n.a. & n.a. \\
\hline 235 & \multirow{3}{*}{ HPHPPH } & Ntyr-Nce-Ntyr-Nhe-Nce-Ntyr & 5872.7613 & $5863.5210[\mathrm{M}+\mathrm{H}]+$ & $95 \%$ & 29 \\
\hline 236 & & Nipr-Nce-Nipr-Npp-Npp-Nbsa & 5913.93 & n.a. & n.a. & n.a. \\
\hline 237 & & Nfu-Nmp-Ndpe-Nmp-Nab-Npip & 6003.944 & n.a. & n.a. & n.a. \\
\hline 238 & \multirow{3}{*}{ HPPHPH } & Nbsa-Nce-Nab-Nbsa-Nab-Nbsa & 6088.12 & $1218.8[\mathrm{M}+5 \mathrm{H}] 5+$ & $95 \%$ & 36 \\
\hline 239 & & Nbu-Namd-Nab-Ndpe-Nab-Ndpe & 5940.0122 & n.a. & n.a. & n.a. \\
\hline 240 & & Nph-Namd-Nme-Nph-Nme-Nipr & 5691.599 & $5683.2041[\mathrm{M}+\mathrm{H}]+$ & $73 \%$ & 17 \\
\hline 241 & \multirow{3}{*}{ PHHPHP } & Nab-Nipr-Nipr-Ncm-Nipr-Nab & 5650.664 & $1413.71[\mathrm{M}+4 \mathrm{H}]$ & $90 \%$ & 51 \\
\hline 242 & & Ncm-Nbn-Nfu-Ncm-Nbn-Namd & 5757.5718 & n.a. & n.a. & n.a. \\
\hline 243 & & Nae-Npip-Ndpe-Nae-Nipr-Ncm & 5824.778 & $1457.29[\mathrm{M}+4 \mathrm{H}]$ & $95 \%$ & 92 \\
\hline 244 & \multirow{3}{*}{ PHPHHP } & Nae-Ncpr-Namd-Ncpr-Ncpr-Nae & 5629.605 & $1408.46[\mathrm{M}+4 \mathrm{H}]$ & $90 \%$ & 27 \\
\hline 245 & & Ncm-Nbu-Nab-Nbu-Ncpr-Ncm & 5677.642 & $1420.31[\mathrm{M}+4 \mathrm{H}]$ & $90 \%$ & 64 \\
\hline 246 & & Nab-Nspe-Nab-Ndpe-Nbu-Npp & 5932.08 & n.a. & n.a. & n.a. \\
\hline 247 & \multirow{3}{*}{$\mathrm{PPHHPH}$} & Nme-Nme-Ntyr-Ntyr-Namd-Ntyr & 5857.804 & n.a. & n.a. & n.a. \\
\hline 248 & & Nce-Nmp-Ncpe-Nipr-Nmp-Ncpe & 5788.7332 & $5775.3403[\mathrm{M}+\mathrm{H}]+$ & $95 \%$ & 23 \\
\hline 249 & & Nae-Namd-Ncpr-Nph-Namd-Nia & 5681.64 & n.a. & n.a. & n.a. \\
\hline 250 & \multirow{3}{*}{ HHPPHH } & Ncpe-Ncpe-Namd-Namd-Ncpe-Ncpe & 5710.6884 & n.a. & n.a. & n.a. \\
\hline 251 & & Nipr-Ntyr-Nae-Nce-Ntyr-Nipr & 5763.7234 & $5756.9761[\mathrm{M}+\mathrm{H}]+$ & $95+\%$ & 40 \\
\hline 252 & & Ncpe-Ncpe-Npp-Nce-Nipr-Nbsa & 5882.92 & n.a. & n.a. & n.a. \\
\hline 253 & \multirow{4}{*}{ PHHHHP } & Nhe-Ncpr-Ncpr-Ncpr-Ncpr-Npp & 5709.731 & $1428.41[\mathrm{M}+4 \mathrm{H}]$ & $90 \%$ & 15 \\
\hline 254 & & Nmp-Ncpr-Ncpr-Nspe-Ncpr-Nae & 5740.7172 & n.a. & n.a. & n.a. \\
\hline 255 & & Namd-Nia-Ntyr-Nia-Nph-Nae & 5760.7661 & $5749.7959[\mathrm{M}+\mathrm{H}]+$ & $93 \%$ & 33 \\
\hline 256 & & Npp-Nspe-Nipr-Nfu-Nfu-Ncm & 5813.75 & n.a. & n.a. & n.a. \\
\hline
\end{tabular}




\section{B. SUPPLEMENTARY DATA}

Anthrax protective antigen (PA63)7 preparation.

a)

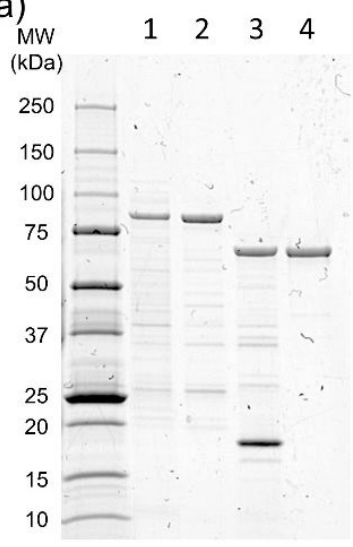

b)

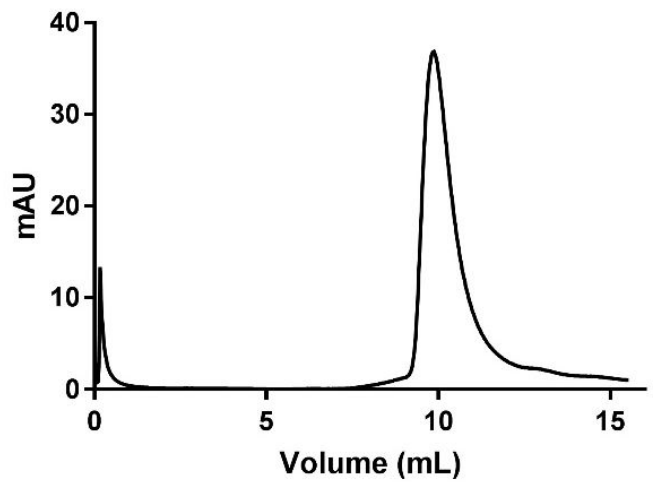

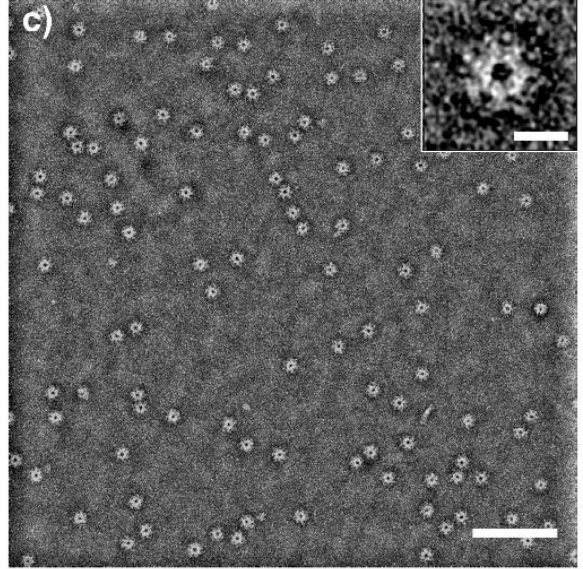

(PA63)7 expression, purification, and characterization. a) SDS-PAGE gel of (PA63) ${ }_{7}$ at different stages of purification. 1) Soluble whole cell lysate containing PA83, 2) PA83 contained in fraction eluted from anion exchange column, 3) PA63 and PA20 after trypsin cleavage of PA83, 4) (PA63) ${ }_{7}$ contained in fraction eluted from second round of anion exchange chromatography. b) UV absorbance spectrum (mAU) versus elution volume $(\mathrm{mL})$ from size exclusion chromatography of Alexa Fluor-647 conjugated (PA63) 7 . c) Survey view of $(\mathrm{PA} 63)_{7}$ by transmission electron microscopy with negative staining. Scale bar $=100 \mathrm{~nm}$. Inset in upper right corner shows higher magnification of a representative particle of $(\mathrm{PA63})_{7}$. Scale bar in inset $=10 \mathrm{~nm}$. 
(a)

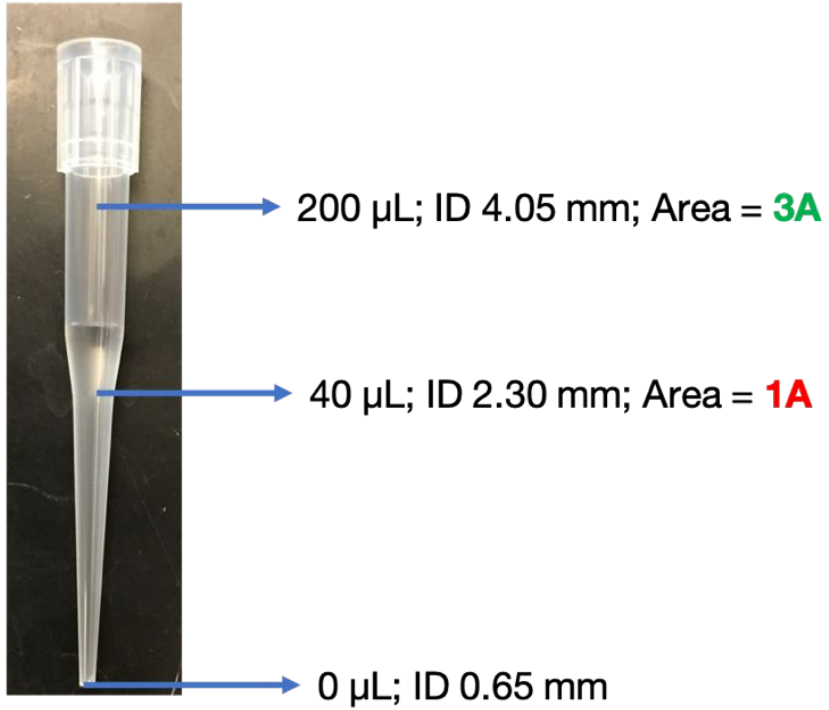

(b)

Aspirate $200 \mu \mathrm{L}$

200 Cycles

Dispense $160 \mu \mathrm{L}$ for $10 \mathrm{~s}$

Aspirate $160 \mu \mathrm{L}$ for $5 \mathrm{~s}$

Wait for $10 \mathrm{~s}$

Dispense $200 \mu \mathrm{L}$

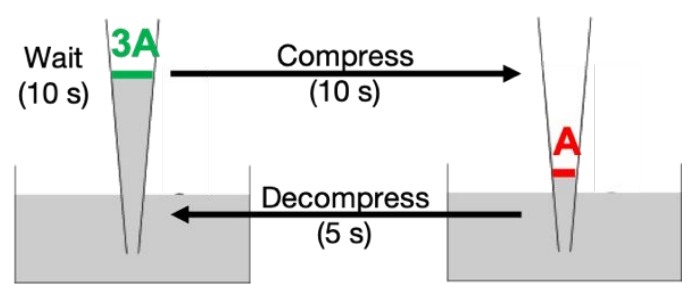

Figure S1. Pipet tip geometry for interfacial compression.

Nanosheets are formed by repeated interfacial compression of a peptoid solution. (a) The inner diameter of a P250 pipet tip defines the air/water interfacial area at different volumes of aspirated liquid. Area compression ratio of 3 can be achieved by changing the volume between the $200 \mu \mathrm{L}$ and $40 \mu \mathrm{L}$ positions. (b) The robotic pipettor is programmed to perform 200 compression cycles. By controlling the aspirate and dispense flow rates $(32 \mu \mathrm{L} / \mathrm{s}$ and $16 \mu \mathrm{L} / \mathrm{s}$, respectively) and the post-aspiration wait time (10 s), the peptoid molecules have enough time to re-populate the newly formed air/water interface during each cycle. 

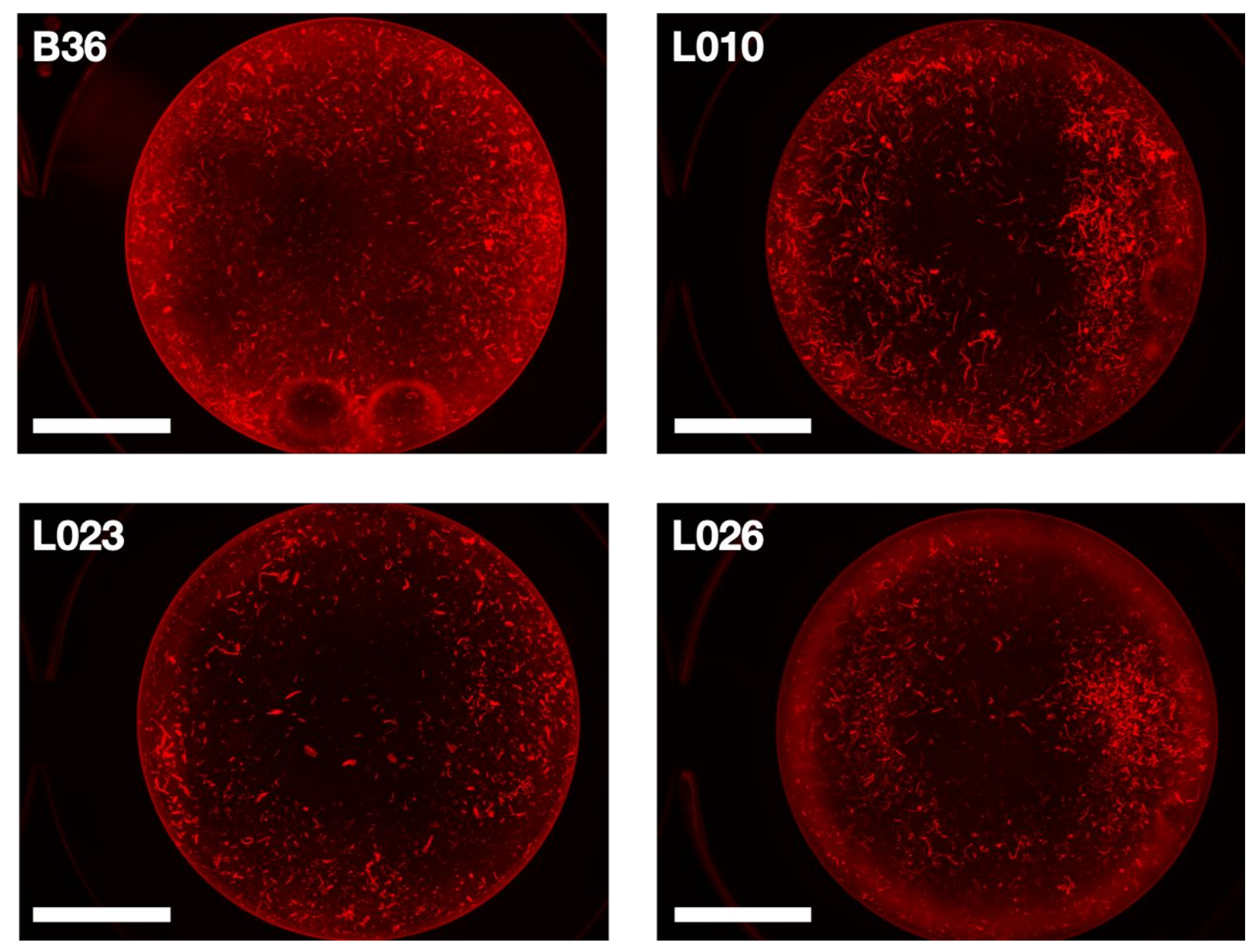

Figure S2. Fluorescence microscopic images of pipet-generated nanosheets.

Representative fluorescence images of nanosheets (loopoid ID's: B36, L010, L023, L026) produced in a 96-well plate produced by the robotic pipetting method. The scale bar is $2 \mathrm{~mm}$. 

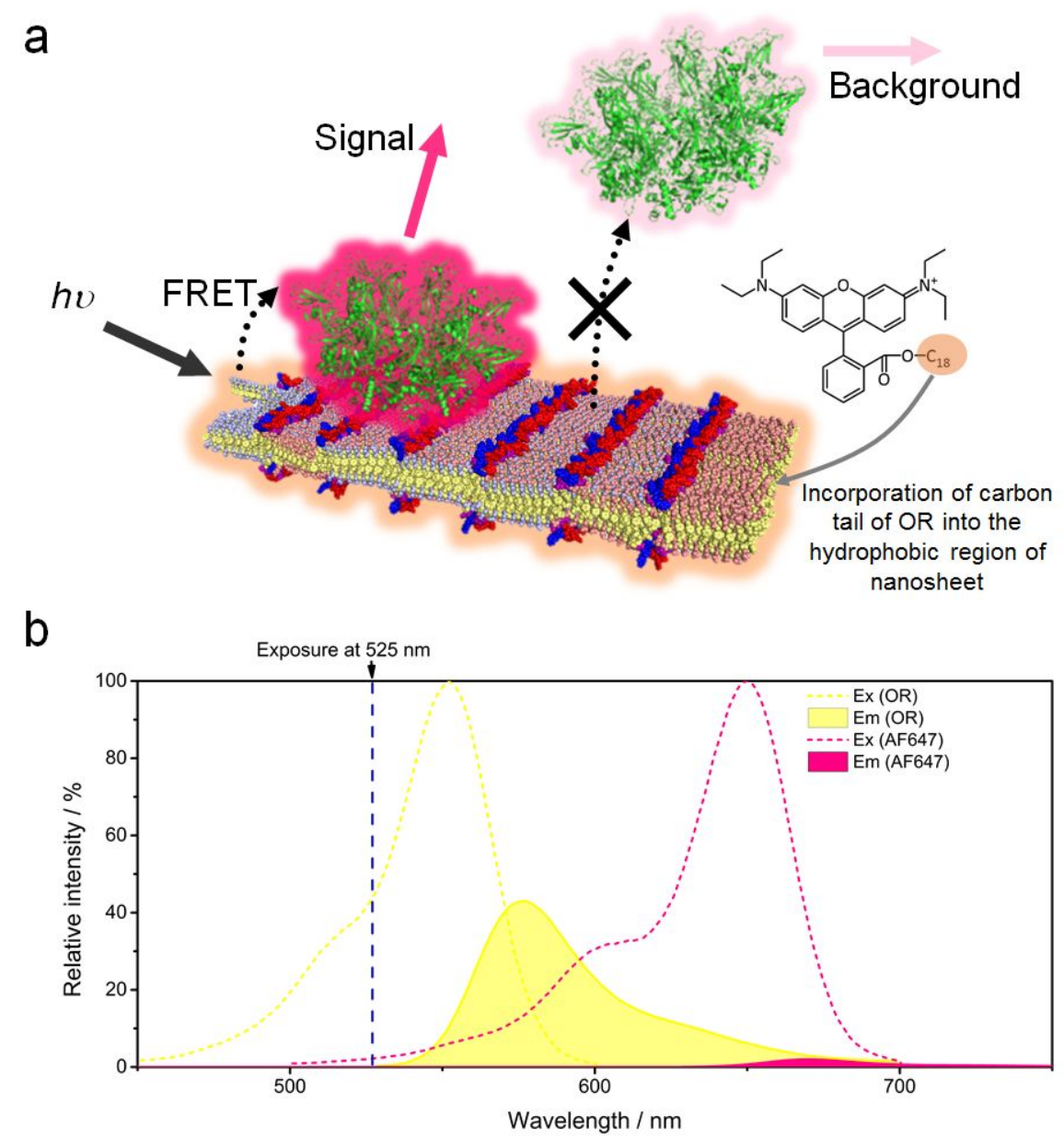

Figure S3. Homogeneous FRET assay for the identification of binding affinity of loopoid nanosheet.

(a) Schematic illustration of FRET assay using the incorporation of octadecyl rhodamine (OR) into nanosheet and Alexa Fluor 647 (AF647)-conjugated protein substrate. Long hydrocarbon chain of OR facilitates insertion into the hydrophobic core of peptoid bilayer. When AF647-conjugated protein is bound on the nanosheet surface, the fluorescence of OR (FRET donor) is transferred into Alexa Fluor 647 (FRET acceptor) via FRET phenomenon. (b) Absorbance (dotted line) and fluorescence (filled area) of OR (yellow) and AF647 (red). The fluorescence emission spectra are normalized to show the relative fluorescence intensities by direct excitation at $525 \mathrm{~nm}$. 


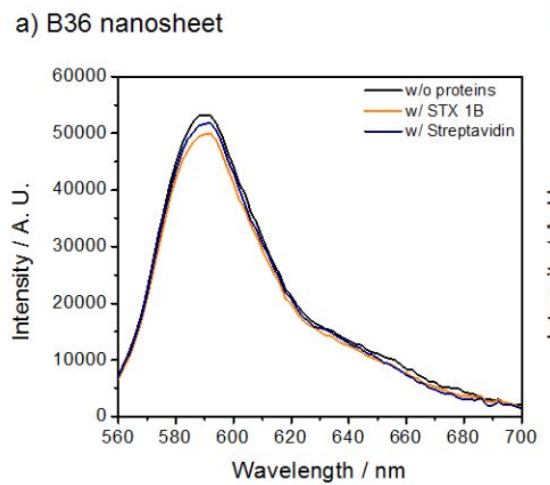

b) Globotriosylated nanosheet

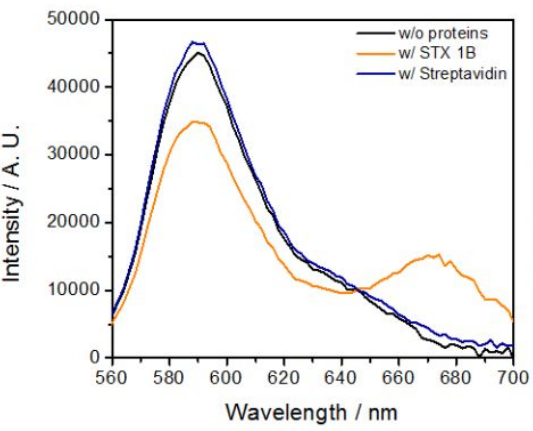

c) Biotinylated nanosheet

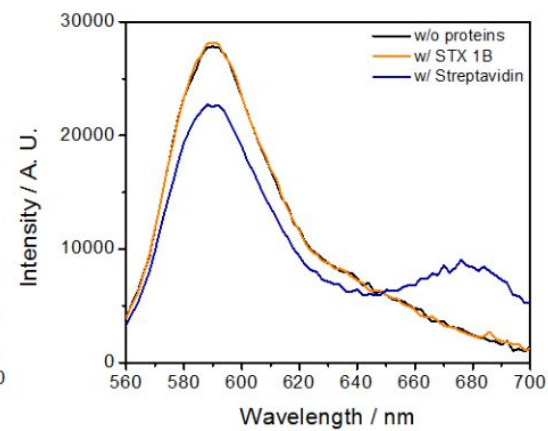

Figure S4. FRET assay to identify protein binding to peptoid nanosheet.

Fluorescence spectra of a) B36, b) globotriosylated, and c) biotinylated nanosheet in the absence (black line) and presence of STX 1B (orange line) and streptavidin (blue line). All spectra were obtained under the excitation wavelength at $525 \mathrm{~nm}$. Globotriose and biotin are binding elements for STX 1B and streptavidin, respectively. 


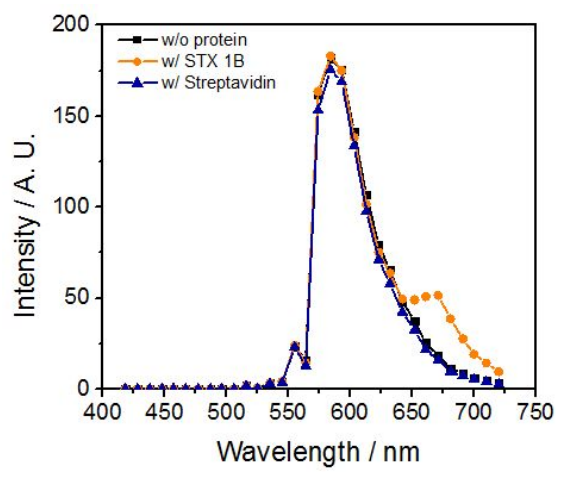

Figure S5. Identification of the origin of FRET signal by confocal microscopy.

Spectra were obtained by spectral scanning of globotriosylated nanosheet in the absence and presence of STX 1B and streptavidin. 
a) FRET ratio ( FRET acceptor/FRET donor)

b) Area-correlated method
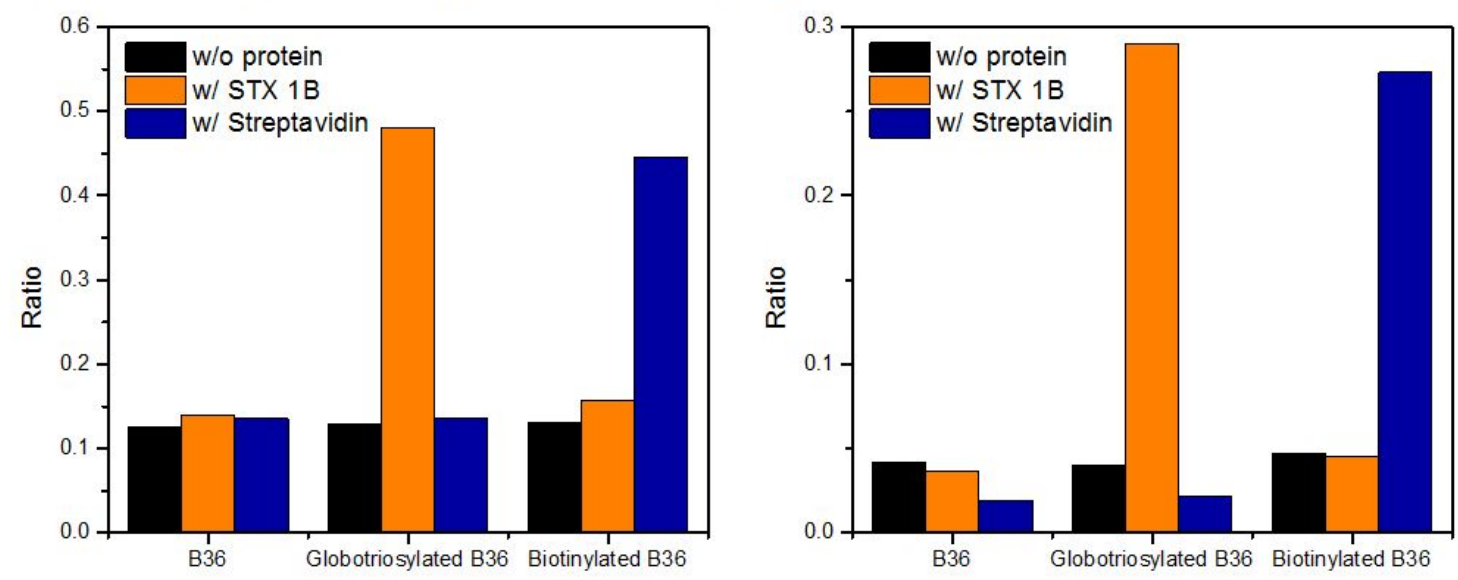

Figure S6. Curve fitting method provides more accurate measure of FRET efficiency.

FRET ratio obtained by a) calculating a simple ratio of donor and acceptor fluorescence intensities $\left(\mathrm{I}_{\mathrm{Alexa647}} / \mathrm{I}_{\mathrm{OR}}\right)$ and $\mathrm{b}$ ) curve fitting fluorescence spectra with a linear combination of donor and acceptor emission spectra and calculating the ratio of the regression coefficients. 


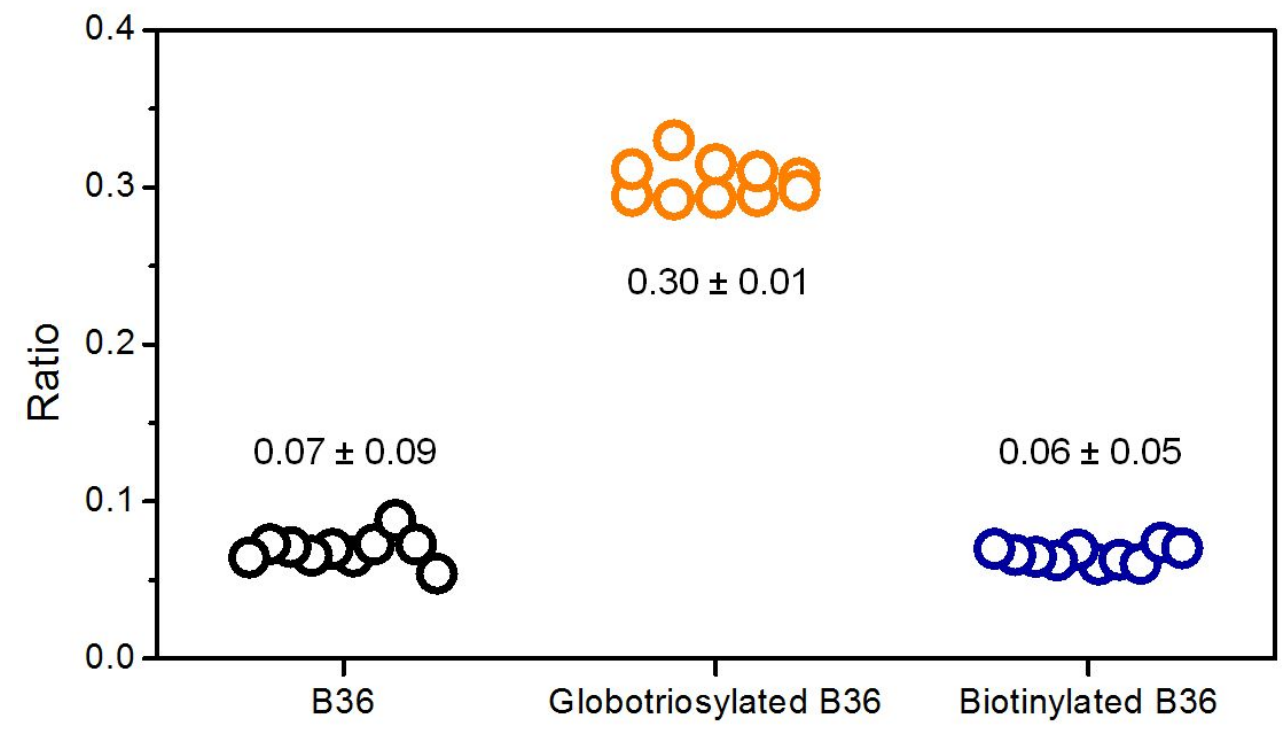

Figure S7. Z-factor calculation validates the reliability of developed FRET assay.

FRET ratio obtained from 10 samples of B36, globotriosylated, and biotinylated nanosheet in the presence of STX 1B. Based on the value of average and standard deviation, Z-factor of our screening assay is approximately 0.73 . 
a) L034 nanosheet
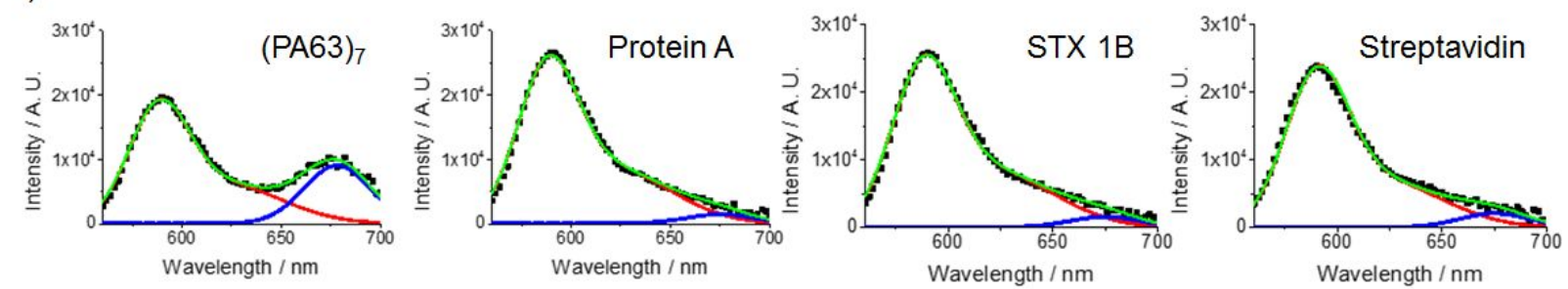

b) TYWWLD nanosheet
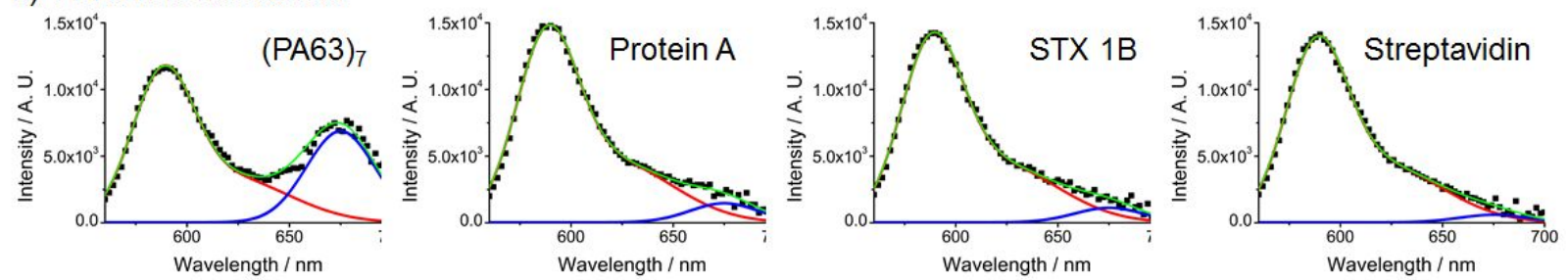

Figure S8. Validation of binding specificity of L034 and TYWWLD nanosheet by FRET assay.

All spectra were obtained under the excitation wavelength at $525 \mathrm{~nm}$ with $2 \mathrm{~nm}$ interval for data acquisition. By curve fitting using Gaussian function-derived donor and acceptor spectra, the emission spectra can be decomposed into OR (red line) and AF647 (blue line) components. Green line represents the sum of OR and AF647 spectra. All nanosheets were prepared by vial-rocking method. Positive FRET signal is clearly observed only in the presence of (PA63) ${ }_{7}$. 


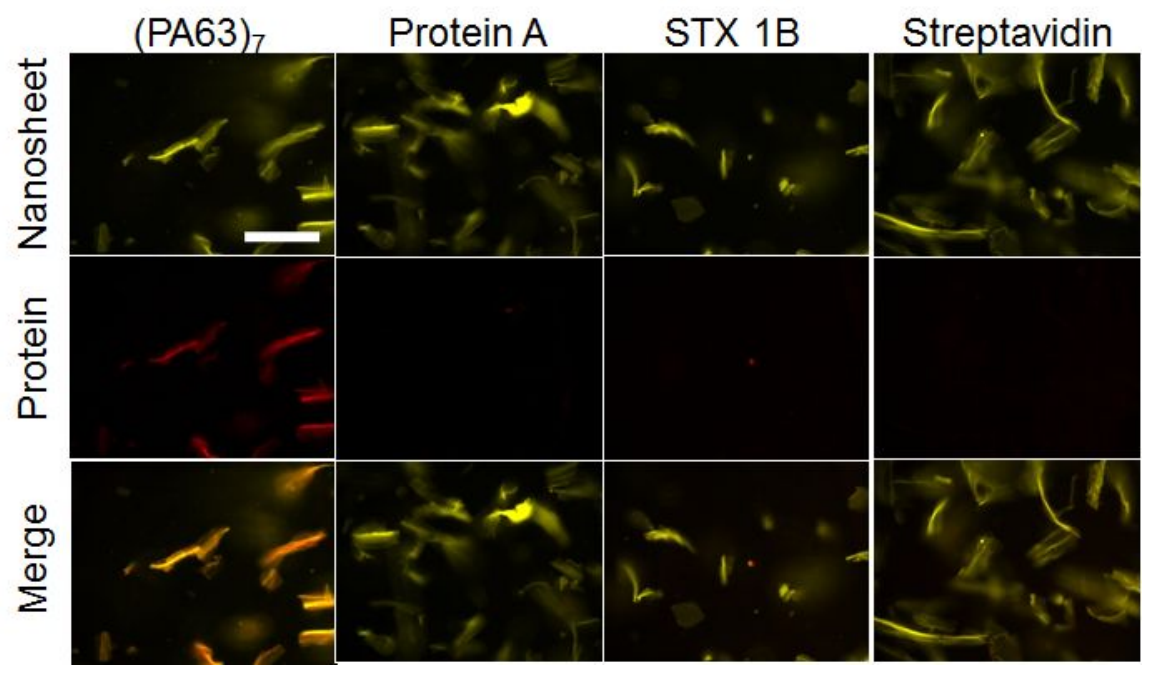

Figure S9. Validation of binding specificity of TYWWLD nanosheet by fluorescent microscopy.

OR-stained nanosheets are visualized with TR filter (yellow color). AF647-conjugated proteins are detected in Cy5 filter (red color). The colocalization of fluorescence signals in the merged image shows the binding specificity of TYWWLD nanosheets. Scale bar represents $100 \mu \mathrm{m}$. All images were acquired with the same magnification. 
Table S2. Comparison of HP pattern and chemical property of monomers between L034 and TYWWLD loop sequences.

\begin{tabular}{|c|c|c|c|c|c|c|c|c|}
\hline ID & HP pattern & & 1st & 2nd & $3 \mathrm{rd}$ & 4th & 5 th & 6 th \\
\hline \multirow{2}{*}{ L034 } & \multirow{2}{*}{ РНнННР } & Monomer & Namd & Ntyr & Ntyr & Ntyr & Ntyr & Namd \\
\hline & & $\begin{array}{l}\text { Chemical } \\
\text { property }\end{array}$ & $\mathrm{Hd}$ & $\mathrm{Ar}$ & $\mathrm{Ar}$ & $\mathrm{Ar}$ & $\mathrm{Ar}$ & $\mathrm{Hd}$ \\
\hline \multirow{2}{*}{ TYWWLD } & \multirow{2}{*}{ РНHНHР } & Monomer & Thr & Tyr & Trp & Trp & Leu & Asp \\
\hline & & $\begin{array}{l}\text { Chemical } \\
\text { property }\end{array}$ & $\mathrm{Hd}$ & $\mathrm{Ar}$ & $\mathrm{Ar}$ & $\mathrm{Ar}$ & $\mathrm{Sa}$ & An \\
\hline
\end{tabular}




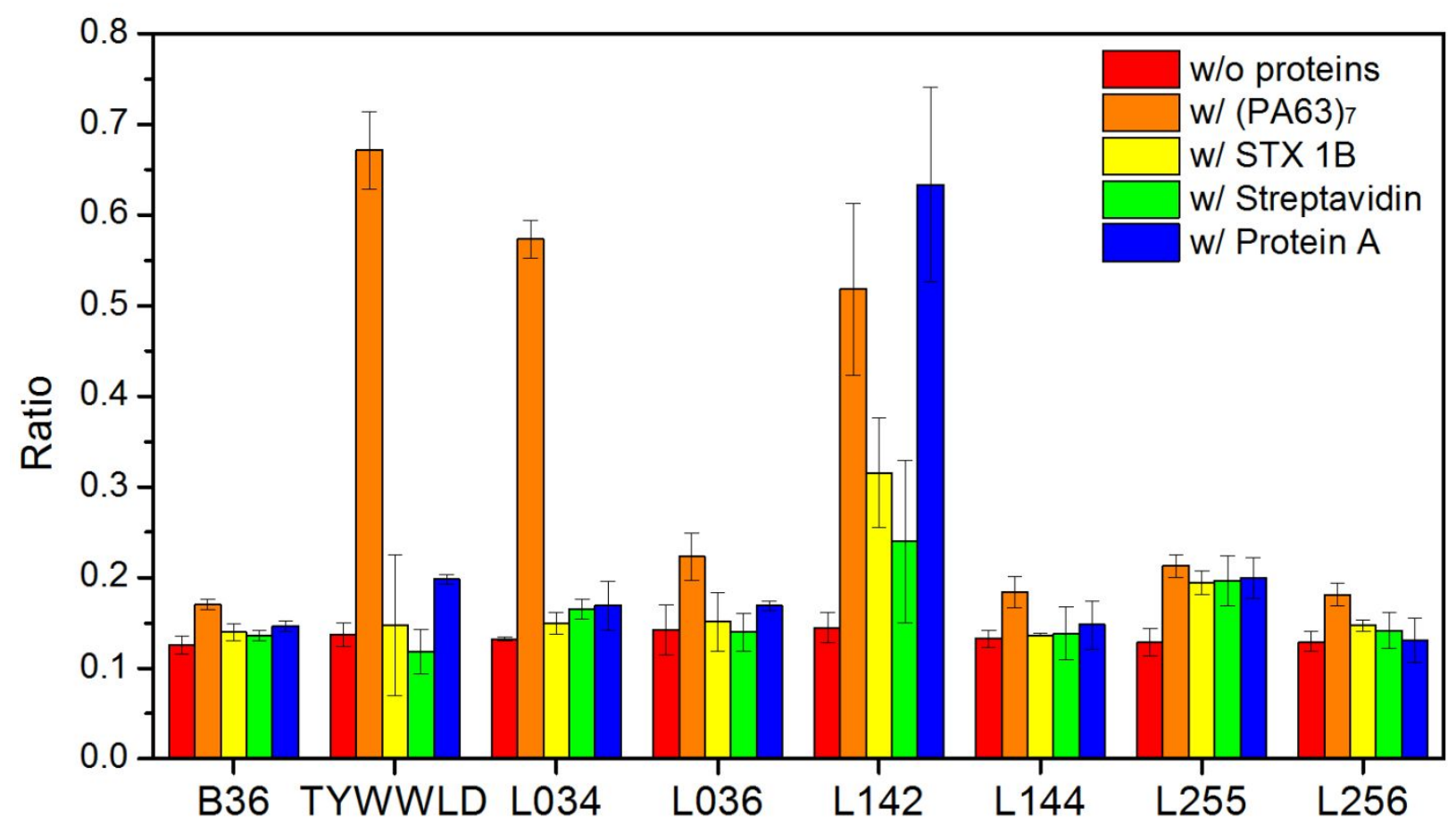

Figure S10. FRET-based validation of protein binding to nanosheets classified as PHHHHP pattern.

Among various nanosheets, only TYWWLD and L034 nanosheets showed high (PA63) 7 binding specificity. L142 represents non-specific binding to multiple target proteins. 

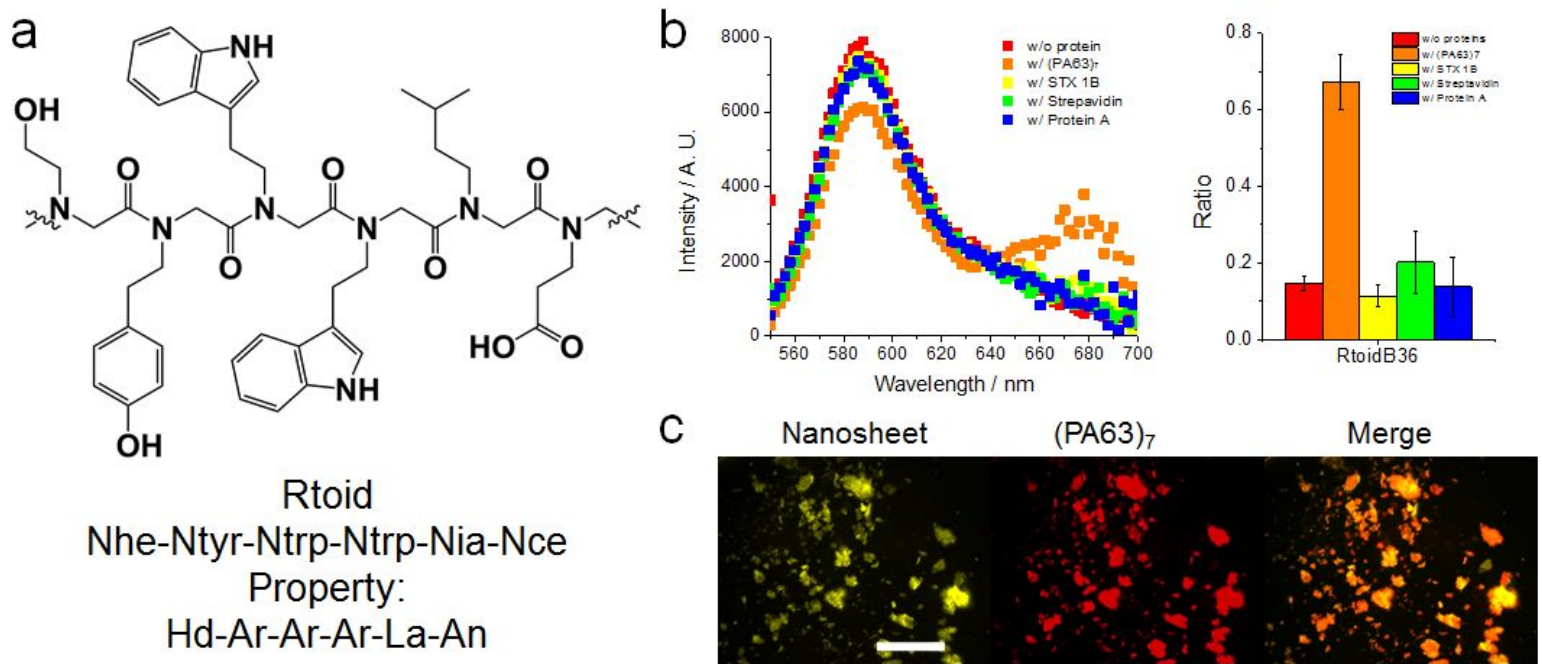

Figure S11. Chemical structure and $\left(\mathrm{PA63}_{7}\right.$ binding specificity of Rtoid.

(a) Sequence and chemical property of Rtoid loop. (b) FRET-based validation of selective (PA63) 7 binding to Rtoid nanosheet. (c) Fluorescent images of Rtoid nanosheet in the presence of (PA63) ${ }_{7}$. 
Table S3. Binding parameters of B36, TYWWLD, and L034 nanosheet measured by bio-layer interferometry.

\begin{tabular}{cccc}
\hline & $\mathrm{Kd}(\mathrm{nM})$ & $\mathrm{k}_{\text {on }}\left(\mathrm{M}^{-1} \mathrm{~s}^{-1}\right)$ & $\mathrm{k}_{\text {dis }}\left(\mathrm{s}^{-1}\right)$ \\
\hline B36 nanosheet & - & - & - \\
\hline TYWWLD nanosheet & 1.59 & $8.71 \times 10^{4}$ & $1.38 \times 10^{-4}$ \\
\hline L034 nanosheet & 1.95 & $4.74 \times 10^{4}$ & $9.24 \times 10^{-5}$ \\
\hline
\end{tabular}

To obtain all binding parameters, all binding curves (Figure 5a) were fitted with 2:1 binding model. No binding observed in B36 nanosheet. 